\title{
ON TANGENT CONE TO SYSTEMS OF INEQUALITIES AND EQUATIONS IN BANACH SPACES UNDER RELAXED CONSTANT RANK CONDITION
}

\author{
E.M. BednarczuK ${ }^{1}$, K.W. Leśniewski ${ }^{2}$ and K.E. RutKowski ${ }^{2,3, *}$
}

\begin{abstract}
Under the relaxed constant rank condition, introduced by Minchenko and Stakhovski, we prove that the linearized cone is contained in the tangent cone (Abadie condition) for sets represented as solution sets to systems of finite numbers of inequalities and equations given by continuously differentiable functions defined on Banach spaces.
\end{abstract}

Mathematics Subject Classification. 47J07, 47J30, 49J27, 49K27, 90C46.

Received October 10, 2019. Accepted January 5, 2021.

\section{INTRODUCTION}

Conditions ensuring the equality between the tangent and the linearized cones to the constraint set are at the core of optimality conditions in constrained optimization. Let $E$ be a Banach space and

$$
\mathcal{F}:=\left\{x \in E \mid h_{i}(x)=0, i \in I_{0}, h_{i}(x) \leq 0, i \in I\right\},
$$

where $h_{i}: E \rightarrow \mathbb{R}, i \in I_{0} \cup I$ are $C^{1}$ functions in a neighbourhood of $x_{0} \in \mathcal{F}$. Sets $I_{0}, I$ are finite, $I_{0} \cup I=$ $\{1,2, \ldots, n\}$, we admit either $I_{0}$ or $I$ to be empty.

Abadie condition has been introduced in [1]. It says that the tangent and linearized cone coincide (see Sect. 6). In [17] it was shown that CRCQ implies Abadie constraint qualification. In the case $I_{0}=\emptyset$ and $h_{i}: \mathbb{R}^{n} \rightarrow \mathbb{R}$, $i \in I$ are convex it was shown in Theorem 3.5 of [20] that Abadie condition is equivalent to the metric regularity of the respective set-valued mapping.

In finite-dimensional settings relationships between constant rank constraint qualification and Abadie condition were investigated in $[6,7]$, and for relationships between relaxed Mangasarian-Fromovitz and Abadie condition see e.g., [18]. When $h_{i}, i \in I_{0} \cup I$ are of class $C^{2}$, the Abadie condition has been investigated in [3]. The survey of the existing finite-dimensional constraint qualification ensuring the Abadie conditions can be found in [27].

Keywords and phrases: Tangent cone, relaxed constant rank condition, Abadie condition, rank theorem, Ljusternik theorem, Lagrange multipliers.

1 Warsaw University of Technology, 00-662 Warszawa, Koszykowa 75, Systems Research Institute of PAS, PAS, 01-447 Warsaw, Newelska 6.

2 Systems Research Institute, PAS, 01-447 Warsaw, Newelska 6.

3 Cardinal Stefan Wyszyński University, 01-815 Warsaw, Dewajtis 5.

* Corresponding author: k.rutkowski@uksw.edu.pl 
In infinite-dimensional case, the most commonly used constraint qualification is Robinson's condition (see $[5,19,26])$ and the relationship to Lagrange multipliers (see $[10,25,30])$.

Due to limitations of applicability of Robinson's condition there exist a number of recent attempts to define other constraint qualification without referring to active index set (for example various forms of cone-continuity properties in [12] and the references therein).

In Proposition 1 of [15], in metric spaces, it was shown that calmness implies Abadie condition. Recently, the Abadie condition on manifolds was investigated in [9].

In the present investigation we consider constrained optimization problems defined on Banach spaces with finite number of constraints. A natural example of such an optimization problem is the projection in Hilbert space onto set of the form (1.1).

The aim of the present paper is to investigate the relationship between the relaxed constant rank constraint qualification (RCRCQ, Def. 6.1), the form of the tangent cone to $\mathcal{F}$ at a given $x_{0} \in \mathcal{F}$ (the Abadie condition) and the existence of Lagrange multipliers to the problem

$$
\begin{array}{ll}
\operatorname{minimize} & h_{0}(x) \\
\text { s.t. } & x \in \mathcal{F},
\end{array}
$$

$h_{0}: E \rightarrow \mathbb{R}$, in Banach space setting.

In the finite-dimensional setting, when $E=\mathbb{R}^{n}$, this question has been discussed in Theorem 1 of [23] (see also [22]). When dealing with the infinite-dimensional case our main tools are Banach space versions of local representation theorem (Thm. 2.5, [2], Thm. 2.1.15), rank theorem (Thm. 4.3, [2], Thm. 2.5.15) and Ljusternik theorem (see e.g., [16], Sect. 0.2.4).

The main results are Theorem 6.5 and Proposition 8.1. The proof of Theorem 6.5 relies mainly on Proposition 5.2. This proposition can be viewed as a variant of the Implicit Function type theorem and relates constant rank condition with functional independence and functional dependence of a finite number of $C^{1}$ functions defined on a Banach space. In Proposition 5.2 we use the concept of functional dependence/independence which generalizes to Banach spaces the concept of Example 2.5.16 in [2] and the proof of Proposition 5.2 is based on rank theorem (Thm. 4.3).

The organization of the paper is as follows. Section 3 is devoted to constant rank condition (CRC). With the help of CRC, in Section 4, we reformulate the classical rank theorem in the case considered (finite number of functions defined on a Banach space). In Section 5 we prove Proposition 5.2 which is the main tool in deriving our main results of Section 6. In Section 6, in Definition 6.1, we define the relaxed constant rank condition (RCRCQ) and we use it to prove the Abadie condition (Thm. 6.5). In Section 7 we discuss other concepts of functional dependence and in Section 8 in Proposition 8.1 we prove the nonemptiness of the Lagrange multipliers set under RCRCQ.

\section{Preliminary FACTS}

We start with some preliminary facts and definitions which will be useful in the sequel.

Let $E$ be a real Banach space and denote $E^{*}$ its dual. Let $\langle\cdot, \cdot\rangle: E^{*} \times E \rightarrow \mathbb{R}$ denote the duality mapping between spaces $E, E^{*}$. We have $\varphi(x)=\langle\varphi, x\rangle$ for all $\varphi \in E^{*}, x \in E$. Let $\|\cdot\|$ denote norm on $E$ and $\|\cdot\|_{*}$ denote norm on $E^{*}$.

Definition 2.1. The closed subspace $H$ of a Banach space $E$ splits or is complemented, if there is a closed subspace $G \subset E$ such that $E=H \oplus G$ (i.e. $E=H+G$ and $H \cap G=\{0\}$ ), where $\oplus$ denotes the direct sum of $H$ and $G$. 
Let $f: U \rightarrow \mathbb{R}^{n}, U \subset E$ open set and $x_{0} \in U$. Fréchet derivative $D f\left(x_{0}\right): U \rightarrow \mathbb{R}^{n}$ is a linear operator defined as

$$
\lim _{\Delta x \rightarrow 0} \frac{\left\|f\left(x_{0}+\Delta x\right)-f\left(x_{0}\right)-D f\left(x_{0}\right) \Delta x\right\|}{\|\Delta x\|}=0 .
$$

Moreover, assuming $f=\left[f_{1}, \ldots, f_{n}\right]$, where $f_{i}: U \rightarrow \mathbb{R}$, we write $D f\left(x_{0}\right)=\left[D f_{1}\left(x_{0}\right), \ldots, D f_{n}\left(x_{0}\right)\right]^{T}$, where $D f_{i}: U \rightarrow \mathbb{R}$.

In the sequel we will use representations of the space $E$ as a direct sum of closed subspaces.

Fact 2.2. Let $V$ be a finite-dimensional subspace of a Banach space $E$. Let $\mathcal{B}=\left\{e_{i}, i=1, \ldots, n\right\}$ be a basis for $V$. Then there exists a basis $\left\{e_{i}^{*}, i=1, \ldots, n\right\} \subset E^{*}$ of $V^{*}$ s.t.

$$
e_{i}^{*}\left(e_{j}\right)=\delta_{i j}:= \begin{cases}1 & \text { if } i=j \\ 0 & \text { if } i \neq j .\end{cases}
$$

Furthermore, for all $x \in V, e_{i}^{*}(x)=0$ for $i=1,2, \ldots, n$ implies that $x=0$ (i.e. $\left\{e_{i}^{*}\right\}_{j=1}^{n}$ is a total family of functionals on $V$ ).

Proof. Let us put $w_{i}\left(e_{j}\right)=\delta_{i j}$ for all $j=1,2, \ldots, n$ then, by Hahn-Banach Theorem, there exists a unique linear functionals $e_{i}^{*} \in E^{*}$, such that $\left.e_{i}^{*}\right|_{V}=w_{i}, i=1,2, \ldots, n$. Let us take any $x \in V$ i.e. $x=\sum_{i=1}^{n} \alpha_{i} e_{i}$ and let us observe that for all $j=1, \ldots, n$ we have

$$
e_{j}^{*}\left(\sum_{i=1}^{n} \alpha_{i} e_{i}\right)=w_{j}\left(\sum_{i=1}^{n} \alpha_{i} e_{i}\right)=\alpha_{j}
$$

and hence, equality $e_{j}^{*}(x)=0$ for all $j=1,2, \ldots, n$ implies that $\alpha_{j}=0$ for all $j=1,2, \ldots, n$. So $\left\{e_{j}^{*}\right\}_{j=1}^{n}$ is a total family of functionals on $V$.

Proposition 2.3. Let $E$ be a Banach space, $f: E \rightarrow \mathbb{R}^{\kappa}, f=\left[f_{1}, \ldots, f_{\kappa}\right], f_{i}: E \rightarrow \mathbb{R}, i=1, \ldots, \kappa, x_{0} \in E$. Assume that functionals $D f_{i}\left(x_{0}\right), i=1, \ldots, \kappa$, are linearly independent. Then $E=E_{1} \oplus E_{2}$, where $E_{2}:=$ ker $D f\left(x_{0}\right), E_{1}:=\operatorname{span}\left\{D f_{1}\left(x_{0}\right)^{*}, \ldots, D f_{\kappa}\left(x_{0}\right)^{*}\right\}$ with $D f_{i}\left(x_{0}\right)^{*}, i=1, \ldots, \kappa$, defined as in Fact 2.2.

Proof. Taking $e_{i}:=D f_{i}\left(x_{0}\right) \in E^{*}, i=1, \ldots, \kappa$, we get $X_{1}:=\operatorname{span}\left\{e_{1}, \ldots, e_{\kappa}\right\}=\operatorname{span}\left\{D f_{1}\left(x_{0}\right), \ldots, D f_{\kappa}\left(x_{0}\right)\right\}$, $\operatorname{dim} X_{1}=\kappa$. By Fact 2.2, there exist vectors $e_{i}^{*}:=D f_{i}\left(x_{0}\right)^{*} \in E^{* *}, i=1, \ldots, \kappa$ satisfying (2.1), i.e.

$$
e_{i}^{*}\left(e_{j}\right)=D f_{i}\left(x_{0}\right)^{*}\left(D f_{j}\left(x_{0}\right)\right)=\left\{\begin{array}{ll}
1 & \text { if } i=j \\
0 & \text { if } i=j
\end{array},\right.
$$

which are linearly independent and $X_{1}^{*}=\operatorname{span}\left\{D f_{i}\left(x_{0}\right)^{*}, 1=1, \ldots, \kappa\right\} \subset E^{* *}$ is a $\kappa$-dimensional subspace of $E^{* *}$.

Since $X_{1}$ is the finite-dimensional space there exists canonical isomorphism (see e.g. By Proposition 10.4 of [14]) eval $_{X_{1}}: X_{1} \rightarrow X_{1}^{* *}$ defined as

$$
\operatorname{eval}_{X_{1}}(v)\left(u^{*}\right)=u^{*}(v) \text { for every } v \in X_{1}, u^{*} \in X_{1}^{*}
$$

and we have $e_{i}^{*}\left(e_{j}\right)=e_{j}\left(e_{i}^{*}\right)=D f_{i}\left(x_{0}\right) D f_{j}\left(x_{0}\right)^{*}$ for all $i, j \in\{1, \ldots, \kappa\}$. By $(2.2)$,

$$
D f\left(x_{0}\right)\left(D f_{i}\left(x_{0}\right)^{*}\right)=v_{i}, \quad v_{i}=[0, \ldots, \underbrace{1}_{i}, \ldots, 0] \in \mathbb{R}^{\kappa} .
$$


Now we show that $E=X_{1}^{*} \oplus \operatorname{ker} D f\left(x_{0}\right)$. For any $x \in E, D f\left(x_{0}\right)(x) \in \mathbb{R}^{\kappa}$, there exist $\alpha_{i}(x) \in \mathbb{R}, i=1, \ldots, \kappa$ such that $D f\left(x_{0}\right)(x)=\sum_{i=1}^{\kappa} \alpha_{i}(x) v_{i}$. Take $m \in X_{1}^{*}, m:=\sum_{i=1}^{\kappa} \alpha_{i}(x) D f_{i}\left(x_{0}\right)^{*}$. By (2.3), we have

$$
D f\left(x_{0}\right)(x-m)=D f\left(x_{0}\right)(x)-D f\left(x_{0}\right)(m)=\sum_{i=1}^{\kappa} \alpha_{i}(x) v_{i}-\sum_{i=1}^{\kappa} \alpha_{i}(x) D f\left(x_{0}\right)\left(D f_{i}\left(x_{0}\right)^{*}\right)=0 .
$$

This shows that $x-m \in \operatorname{ker} D f\left(x_{0}\right)$ which proves the assertion with $E_{1}:=X_{1}^{*}$ and $E_{2}:=\operatorname{ker} D f\left(x_{0}\right)$.

In the sequel we will make a frequent use of the following observation.

Lemma 2.4. Let $E$ be a Banach space and $f: E \rightarrow \mathbb{R}^{p}, f=\left[f_{1}, \ldots, f_{p}\right], f_{i}: E \rightarrow \mathbb{R}, i=1, \ldots, p$ be $C^{1}$ functions in some neighbourhood of $x_{0} \in E$. If $D f_{j}\left(x_{0}\right), j=1, \ldots, p$ are linearly independent, then there exists a neighbourhood $U_{0}\left(x_{0}\right)$ such that elements $D f_{j}(x), j=1, \ldots, p$ are linearly independent for all $x \in U_{0}\left(x_{0}\right)$.

Proof. By assumption, $D f_{i}\left(x_{0}\right), i=1, \ldots, p$ is a basis of $\operatorname{span}\left\{D f_{i}\left(x_{0}\right), i=1, \ldots, p\right\}$. By Fact 2.2 , there exist $\left(e_{1}\left(x_{0}\right)\right)^{*}, \ldots,\left(e_{p}\left(x_{0}\right)\right)^{*}$, such that

$$
\left(e_{j}\left(x_{0}\right)\right)^{*}\left(D f_{i}\left(x_{0}\right)\right)=\left\{\begin{array}{cc}
1 & i=j \\
0 & i \neq j
\end{array}, \quad i, j \in\{1, \ldots, p\}\right.
$$

$\operatorname{Put}\left(e\left(x_{0}\right)\right)^{*}:=\left[\left(e_{1}\left(x_{0}\right)\right)^{*}, \ldots,\left(e_{p}\left(x_{0}\right)\right)^{*}\right]^{T}$ and $G(x):=\left[\left(e\left(x_{0}\right)\right)^{*} D f_{1}(x), \ldots,\left(e\left(x_{0}\right)\right)^{*} D f_{p}(x)\right], x \in E$. By $(2.4)$,

$$
\operatorname{det} G\left(x_{0}\right)=\operatorname{det}\left[\left(e\left(x_{0}\right)\right)^{*} D f_{1}\left(x_{0}\right), \ldots,\left(e\left(x_{0}\right)\right)^{*} D f_{p}\left(x_{0}\right)\right]=1
$$

and by the continuity of $D f_{i}$, there exists a neighbourhood $U_{0}(x)$ such that $\operatorname{det} G(x)>0$ for $x \in U_{0}\left(x_{0}\right)$. Consequently, for any $x \in U_{0}\left(x_{0}\right)$,

$$
\begin{aligned}
& \alpha_{1} D f_{1}(x)+\cdots+\alpha_{p} D f_{p}(x)=0 \Rightarrow\left(e\left(x_{0}\right)\right)^{*}\left(\alpha_{1} D f_{1}(x)+\ldots \alpha_{p} D f_{p}(x)\right)=0 \\
& \Longleftrightarrow \alpha_{1}\left(e\left(x_{0}\right)\right)^{*} D f_{1}(x)+\cdots+\alpha_{p}\left(e\left(x_{0}\right)\right)^{*} D f_{p}(x)=0 \Longleftrightarrow \alpha_{1}, \ldots, \alpha_{p}=0 .
\end{aligned}
$$

Therefore, for any $x \in U_{0}\left(x_{0}\right), D f_{i}(x), i=1, \ldots, p$ are linearly independent.

Theorem 2.5. ([2], Thm. 2.5.14. Local Representation Theorem) Let $f: U \rightarrow \mathbb{R}^{n}$ be of class $C^{r}, r \geq 1$ in a neighbourhood of $x_{0} \in U, U \subset E$ open set. Let $F_{1}$ be closed split image of $D f\left(x_{0}\right)$ with closed complement $F_{2}$. Suppose that $D f\left(x_{0}\right)$ has split kernel $E_{2}=\operatorname{ker} D f\left(x_{0}\right)$ with closed complement $E_{1}$. Then there are open sets $U_{1} \subset U \subset E_{1} \oplus E_{2}$ and $U_{2} \subset F_{1} \oplus E_{2}, x_{0} \in U_{2}$ and $a C^{r}$ diffeomorphism $\psi: U_{2} \rightarrow U_{1}$ such that $(f \circ \psi)(u, v)=$ $(u, \eta(u, v))$ for any $(u, v) \in U_{1}$, where $u \in E_{1}, v \in E_{2}$ and $\eta: U_{2} \rightarrow E_{2}$ is a $C^{r}$ map satisfying $D \eta\left(\psi^{-1}\left(x_{0}\right)\right)=0$.

Remark 2.6. If dim range $D f(x)=k$ for all $x$ in some neighbourhood $U^{\prime}\left(x_{0}\right)$, then, by Inverse Function Theorem (see e.g. [2], Thm. 2.5.7), there exists an invertible function $\Psi^{\prime}: U^{\prime}\left(x_{0}\right) \rightarrow U$ such that $f \circ \Psi^{\prime}$ depends on $k$ variables.

\section{Constant Rank COndition (CRC)}

In the present section we recall basic facts related to constant rank condition for $C^{1}$ functions. By rank of $A$ (where $A$ is a finite set of elements of vector space) we will understand the cardinality of maximally linearly independent subset of elements of $A$.

Definition 3.1. Let $f_{i}: U \rightarrow \mathbb{R}, i=1, \ldots, \kappa$ be $C^{1}$ functions in a neighbourhood of $x_{0} \in U, U \subset E$ open set. We say that constant rank condition $(\mathrm{CRC})$ holds at $x_{0}$ if there exists a neighbourhood $V\left(x_{0}\right)$ such that

$$
\operatorname{rank}\left\{D f_{i}\left(x_{0}\right), i=1, \ldots, \kappa\right\}=\text { const }=\operatorname{rank}\left\{D f_{i}(x), i=1, \ldots, \kappa\right\}
$$


for all $x \in V\left(x_{0}\right)$. We also admit const $=0$, which corresponds to the case $D f_{i}\left(x_{0}\right)=0, i=1, \ldots, \kappa$.

The constant rank condition appears in the literature ([2], p. 127, [11], p. 47 and [29], p. 503 (under the name same rank). ${ }^{1}$

Let us note that, when $f=\left[f_{1}, \ldots, f_{n}\right], f_{i}: U \rightarrow \mathbb{R}, i=1, \ldots, n$ are of class $C^{1}, U \subset E$ open, and $\operatorname{rank}\left\{D f_{i}\left(x_{0}\right), i=1, \ldots, n\right\}=k$, then $\operatorname{dim} F_{1}=\operatorname{dim} D f\left(x_{0}\right)(E)=k$, where

$$
D f\left(x_{0}\right) y=\left[\begin{array}{c}
\left\langle D f_{1}\left(x_{0}\right), y\right\rangle \\
\vdots \\
\left\langle D f_{n}\left(x_{0}\right), y\right\rangle
\end{array}\right], \quad y \in E
$$

Consequently, $F_{1}, \operatorname{dim} F_{1}=k$, splits $\mathbb{R}^{n}$ and $F_{2}$ is a closed complement of $F_{1}$, $\operatorname{dim} F_{2}=n-k$.

Moreover, for any $e \in B(0, \delta) \subset E$,

$$
\left\|D f\left(x_{0}\right) e\right\|:=\left\|\begin{array}{c}
\left\langle D f_{1}\left(x_{0}\right), e\right\rangle \\
\vdots \\
\left\langle D f_{n}\left(x_{0}\right), e\right\rangle
\end{array}\right\|_{1}=\sum_{i=1}^{n}\left|\left\langle D f_{i}\left(x_{0}\right), e\right\rangle\right| \leq\|e\| \sum_{i=1}^{n}\left\|D f_{i}\left(x_{0}\right)\right\|_{*}<\varepsilon,
$$

whenever $\delta<\frac{\varepsilon}{\left\|D f_{i}\left(x_{0}\right)\right\|_{*}}$, i.e., $D f\left(x_{0}\right)$ is continuous, and $E_{2}:=\operatorname{ker} D f\left(x_{0}\right)=\left\{h \in E \mid D f\left(x_{0}\right) h=0\right\}$ is a closed subspace of $E$. By Proposition 2.3, its complement $E_{1}$ is a closed subspace of $E$, moreover, $\operatorname{dim} E_{1}=k$.

\section{RANK THEOREM UNDER CRC}

In this section we reformulate the classical rank theorem, Theorem 4.3, under CRC condition.

Let $x_{0} \in E$ and $E_{2}=\operatorname{ker} D f\left(x_{0}\right)$. By $E_{1}$ we denote its closed complement (see Prop. 2.3).

Lemma 4.1. Let $E$ be a Banach space. Let $f_{i}: U \rightarrow \mathbb{R}, i=1, \ldots, \kappa, U \subset E$ open, be $C^{1}$ functions in a neighbourhood of $x_{0} \in U$ and let $f=\left[f_{1}, \ldots, f_{\kappa}\right]$. Assume that functionals $D f_{i}(x), i=1, \ldots, \kappa$, are linearly independent for $x$ from a neighbourhood $U_{0}\left(x_{0}\right)$. Then for any $x \in U_{0}\left(x_{0}\right)$, the vectors

$$
e^{i}(x):=D f(x) D f_{i}\left(x_{0}\right)^{*}, \quad i=1, \ldots \kappa,
$$

form a basis in $\mathbb{R}^{\kappa}$, where $D f_{i}\left(x_{0}\right)^{*} \in E, i=1, \ldots \kappa$, (see Fact 2.2) are such that

$$
D f_{i}\left(x_{0}\right)^{*}\left(D f_{j}\left(x_{0}\right)\right)= \begin{cases}1 & \text { if } i=j \\ 0 & \text { if } i \neq j\end{cases}
$$

Proof. The existence of $D f_{i}\left(x_{0}\right)^{*}, i=1, \ldots, \kappa$ is ensured by Fact 2.2. Since $D f_{1}\left(x_{0}\right), \ldots, D f_{\kappa}\left(x_{0}\right)$ are linearly independent, by Fact $2.2, D f_{1}\left(x_{0}\right)^{*}, \ldots, D f_{\kappa}\left(x_{0}\right)^{*}$ are linearly independent.

Let $E_{1}:=\operatorname{span}\left\{D f_{1}\left(x_{0}\right)^{*}, \ldots, D f_{\kappa}\left(x_{0}\right)^{*}\right\}$. First, let us show that for all $x \in U_{0}\left(x_{0}\right)$ the mapping $\left.D f(x)\right|_{E_{1}}$ : $E_{1} \rightarrow D f(x)(E)$ is an injection.

We start this by showing that for $x=x_{0}$ this mapping is an injection. Indeed, suppose that there exists $e_{1}, e_{2} \in E_{1}, e_{1} \neq e_{2}$ such that $D f\left(x_{0}\right)\left(e_{1}\right)=D f\left(x_{0}\right)\left(e_{2}\right)$. Then $e_{1}-e_{2} \in E_{1}$ since $E_{1}$ is a linear space and at the same time $e_{1}-e_{2} \in E_{2}$. This contradicts the fact that $e_{1} \neq e_{2}$.

By assumption, for any $x \in U_{0}\left(x_{0}\right)$ there exists a linear isomorphism

$$
L_{x}: E_{1} \rightarrow \operatorname{span}\left\{D f_{1}(x)^{*}, \ldots, D f_{\kappa}(x)^{*}\right\}
$$

\footnotetext{
${ }^{1}$ Let us note that the same terminology (constant rank condition) has been already used in [4] (Def. 1) and is stronger than that proposed in Definition 3.1.
} 
defined as $L_{x}\left(D f_{j}\left(x_{0}\right)^{*}\right)=D f_{j}(x)^{*}, j=1, \ldots, \kappa$.

Hence, for any $x \in U_{0}\left(x_{0}\right), E_{1}=L_{x}^{-1}\left(\operatorname{span}\left\{D f_{1}(x)^{*}, \ldots, D f_{\kappa}(x)^{*}\right\}\right)$ and $\left.D f(x)\right|_{E_{1}}: E_{1} \rightarrow D f(x)(E)$ is an injection (by injectivity of composition of two injective mappings).

Take any $x \in U_{0}\left(x_{0}\right)$ and $\alpha_{i}(x), i=1, \ldots, \kappa$ such that

$$
\alpha_{1}(x) e^{1}(x)+\cdots+\alpha_{\kappa}(x) e^{\kappa}(x)=0 .
$$

By injectivity of $\left.D F(x)\right|_{E_{1}}, k \in \mathbb{N}$ and by linear independence of $D f_{i}\left(x_{0}\right)^{*}, i=1, \ldots, \kappa$, for all $k$ we are getting the lineary independece of $e^{i}(x), i=1,2, \ldots, \kappa$ for $x \in U_{0}\left(x_{0}\right)$.

Thus, $e^{j}(x), j=1, \ldots, \kappa$, form a basis in $\mathbb{R}^{\kappa}$ for any $x \in U_{0}\left(x_{0}\right)$.

Proposition 4.2. Let $E$ be a Banach space. Let $f_{i}: U \rightarrow \mathbb{R}, i=1, \ldots, \kappa, U \subset E$ open, be $C^{1}$ functions in a neighbourhood of $x_{0} \in U$. Then the following statements are equivalent:

(i) CRC holds at $x_{0}$

(ii) the mapping $\left.D f(x)\right|_{E_{1}}: E_{1} \rightarrow D f(x)(E)$ is an isomorphism for $x$ in some neighbourhood of $x_{0}$.

Proof. (i) $\Longrightarrow$ (ii). Let CRC hold at $x_{0}$ with a neighbourhood $V\left(x_{0}\right)$. Let $\operatorname{rank}\left\{D f_{i}\left(x_{0}\right), i=1, \ldots, \kappa\right\}=k$. By Lemma 2.4, there exist indices $i_{1}, \ldots, i_{k} \subset\{1, \ldots, \kappa\}$, such that $i_{j} \neq i_{l}$ for $j \neq l$, and a neighbourhood $U_{0}\left(x_{0}\right)$ such that

$$
D f_{i_{1}}(x), \ldots, D f_{i_{k}}(x)
$$

form a maximally linearly independent subset of $\left\{D f_{j}(x), j=1, \ldots, \kappa\right\}, x \in U_{0}\left(x_{0}\right)$.

Let $f^{1}(x):=\left[f_{i_{1}}(x), \ldots, f_{i_{k}}(x)\right]$. Clearly, $\operatorname{ker} D f\left(x_{0}\right)=\operatorname{ker} D f^{1}\left(x_{0}\right)$, where $\operatorname{ker} D f^{1}\left(x_{0}\right)=\{h \in E$ | $\left.D f^{1}\left(x_{0}\right) h=0\right\}$. By Proposition 2.3,

$$
E_{1}=\operatorname{span}\left\{D f_{i_{1}}\left(x_{0}\right)^{*}, \ldots, D f_{i_{k}}\left(x_{0}\right)^{*}\right\}
$$

and $\operatorname{dim} E_{1}=k$. By CRC, $\operatorname{dim}(D f(x)(E))=k$ for all $x \in U_{0}\left(x_{0}\right)$.

Since $D f_{i_{1}}\left(x_{0}\right), \ldots, D f_{i_{k}}\left(x_{0}\right)$ are linearly independent, the mapping $\left.D f^{1}(x)\right|_{E_{1}}: E_{1} \rightarrow D f^{1}\left(x_{0}\right)(E)$ is an injection for all $x \in U\left(x_{0}\right)$ (see proof of Lem. 4.1).

Now we discuss the surjectivity of $\left.D f(x)\right|_{E_{1}}: E_{1} \rightarrow D f(x)(E)$ in a neighbourhood of $x_{0}$. To this aim we note that it is enough to investigate the surjectivity of $\left.D f^{1}(x)\right|_{E_{1}}: E_{1} \rightarrow D f^{1}(x)(E)$.

Let us note that $D f(x) e, e \in E_{1}$ is fully determined by $D f^{1}(x) e$. To see this take $e \in E_{1}$. Then $e=$ $\sum_{j=1}^{k} \lambda_{j}\left(D f_{i_{j}}\left(x_{0}\right)\right)^{*}$, where $\lambda_{j} \in \mathbb{R}, j=1, \ldots, k$. For any $x \in U_{0}\left(x_{0}\right)$ we have

$$
D f^{1}(x) e=\left[D f_{i_{l}}(x) e\right]_{l=1}^{k}=\left[\sum_{j=1}^{k} \lambda_{j} D f_{i_{l}}(x) D f_{i_{j}}\left(x_{0}\right)^{*}\right]_{l=1}^{k} .
$$

Again, by Lemma 2.4, D $f_{l}(x)^{*}, l \in\{1, \ldots, \kappa\} \backslash\left\{i_{1}, \ldots, i_{k}\right\}$ depend linearly on $D f_{i_{1}}(x)^{*}, \ldots, D f_{i_{k}}(x)^{*}, x \in$ $U_{0}\left(x_{0}\right) \cap V\left(x_{0}\right)$. We have

$$
D f_{l}(x) e=\sum_{j=1}^{k} \alpha_{j}^{l}(x) D f_{i_{j}}(x) e
$$


where $\alpha_{j}^{l}(x) \in \mathbb{R}, j \in 1, \ldots, k, l \in\{1, \ldots, \kappa\} \backslash\left\{i_{1}, \ldots, i_{k}\right\}, x \in U_{0}\left(x_{0}\right) \cap V\left(x_{0}\right)$ and

$$
D f_{l}(x)=\sum_{j=1}^{k} \alpha_{j}^{l}(x) D f_{i_{j}}(x) .
$$

Now we show the surjectivity of $\left.D f(x)\right|_{E_{1}}: E_{1} \rightarrow D f(x)(E)$ for $x$ in some neighbourhood of $x_{0}$. By Lemma 4.1, there exists a neighbourhood $U_{1}\left(x_{0}\right)$ such that the vectors

$$
e^{j}(x):=D f^{1}(x) D f_{i_{j}}\left(x_{0}\right)^{*}, \quad j=1, \ldots, k
$$

form a basis in $\mathbb{R}^{k}$. Let $x \in U_{0}\left(x_{0}\right) \cap U_{1}\left(x_{0}\right) \cap V\left(x_{0}\right), g \in D f(x)(E)$ and for $l \in\{1, \ldots, \kappa\}$ let us denote by $g_{l}$ its $l$-th component. By (4.2), we have $g_{l}=\sum_{j=1}^{k} \alpha_{j}^{l}(x) g_{i_{h}}, l \in\{1, \ldots, \kappa\} \backslash\left\{i_{1}, \ldots, i_{k}\right\}$ and, moreover

$$
\left[\begin{array}{c}
g_{i_{1}} \\
\vdots \\
g_{i_{k}}
\end{array}\right]=\sum_{j=1}^{k} \beta_{j}(x) e^{j}(x)
$$

for some $\beta_{j}(x) \in \mathbb{R}, j=1, \ldots, k$. Hence,

$$
\left[\begin{array}{c}
g_{i_{1}} \\
\vdots \\
g_{i_{k}}
\end{array}\right]=\sum_{j=1}^{k} \beta_{j}(x) D f^{1}(x) D f_{i_{j}}\left(x_{0}\right)^{*}=D f^{1}(x)\left(\sum_{j=1}^{k} \beta_{j}(x) D f_{i_{j}}\left(x_{0}\right)^{*}\right)
$$

And, for $l \in\{1, \ldots, \kappa\} \backslash\left\{i_{1}, \ldots, i_{k}\right\}$,

$$
g_{l}=\sum_{h=1}^{k} \alpha_{h}^{l}(x) D f_{i_{h}}(x)\left(\sum_{j=1}^{k} \beta_{j}(x) D f_{i_{j}}\left(x_{0}\right)^{*}\right) .
$$

Observe that $\sum_{j=1}^{k} \beta_{j}(x) D f_{i_{j}}\left(x_{0}\right)^{*} \in E_{1}$, and hence $\left.D f(x)\right|_{E_{1}}: E_{1} \rightarrow D f(x)(E)$ is surjective for $x \in$ $U_{0}\left(x_{0}\right) \cap U_{1}\left(x_{0}\right) \cap V\left(x_{0}\right)$. Since $\left.D f(x)\right|_{E_{1}}: E_{1} \rightarrow D f(x)(E)$ is surjection and injection between finitedimensional spaces, it is a (linear) isomorphism.

(ii) $\Longrightarrow$ (i) We have $E_{2}=\operatorname{ker} D f\left(x_{0}\right)=\left\{h \in E \mid D f\left(x_{0}\right) h=0\right\}$ and let $k=\operatorname{rank}\left\{D f_{1}\left(x_{0}\right), \ldots, D f_{\kappa}\left(x_{0}\right)\right\}$. There exists $i_{1}, \ldots, i_{k} \subset\{1, \ldots, \kappa\}$ such that the system $D f_{i_{1}}\left(x_{0}\right), \ldots, D f_{i_{k}}\left(x_{0}\right)$ forms a maximally linearly independent subset of set $\left\{D f_{1}\left(x_{0}\right), \ldots, D f_{\kappa}\left(x_{0}\right)\right\}$. Moreover,

$$
E_{1}=E_{2}^{\perp}=\operatorname{span}\left\{D f_{1}\left(x_{0}\right)^{*}, \ldots, D\left(f_{\kappa}\left(x_{0}\right)\right)^{*}\right\}=\operatorname{span}\left\{D f_{i_{1}}\left(x_{0}\right)^{*}, \ldots, D f_{i_{k}}\left(x_{0}\right)^{*}\right\} .
$$

Since $f_{i_{1}}, \ldots, f_{i_{k}}$ are of class $C^{1}$ there exists a neighbourhood $U_{0}\left(x_{0}\right)$ such that $D f_{i_{1}}(x), \ldots, D f_{i_{k}}(x)$ are linearly independent.

By assumption $\operatorname{dim} D f(x)(E)=k$ for all $x$ in a neighbourhood $V_{1}\left(x_{0}\right) \subset U_{0}\left(x_{0}\right)$. Thus $\left\{D f_{i_{1}}(x)^{*}, \ldots, D f_{i_{k}}(x)^{*}\right\}$ forms a maximally linearly independent subset of set $\left\{D f_{1}(x)^{*}, \ldots, D f_{\kappa}(x)^{*}\right\}, x \in V_{1}\left(x_{0}\right)$. Hence CRC holds for functions $f_{i}: E \rightarrow \mathbb{R}, i=1, \ldots, \kappa$ at $x_{0}$ with neighbourhood $V_{1}\left(x_{0}\right)$.

In view of Proposition 4.2, Theorem 2.5.15 of [2] (for the finite-dimensional case see [21, 29]) takes the following form in the case considered in the present paper.

Theorem 4.3 (Rank theorem under CRC). Let $E$ be a Banach space. Let $x_{0} \in U$, where $U$ is an open subset of $E$ and $f: U \rightarrow \mathbb{R}^{\kappa}, f=\left[f_{1}, \ldots, f_{\kappa}\right], f_{i}: E \rightarrow \mathbb{R}, i=1, \ldots, n$ be $C^{1}$ functions in a neighbourhood of $x_{0}$. Assume 
that $C R C$ holds at $x_{0}$ with a neighbourhood $V\left(x_{0}\right)$ and the constant rank $k$. As previously, let $E_{2}=\operatorname{ker} D f\left(x_{0}\right)$, and let $E_{1}$ be its closed complement. Then there exist open sets $U_{1} \subset \mathbb{R}^{k} \oplus E_{2}, U_{2} \subset E, V_{1} \subset \mathbb{R}^{\kappa}, V_{2} \subset \mathbb{R}^{k} \oplus E_{2}$ and diffeomorphisms of class $C^{1}, \varphi: V_{1} \rightarrow V_{2}$ and $\psi: U_{1} \rightarrow U_{2}, x_{0}=\left(x_{01}, x_{02}\right) \in U_{2} \subset U \subset E_{1} \oplus E_{2}$, i.e. $x_{01} \in E_{1}, x_{02} \in E_{2}, f\left(x_{0}\right) \in V_{1}$ satisfying

$$
(\varphi \circ f \circ \psi)(w, e)=(w, 0), \quad \text { where } w \in E_{1}, e \in E_{2}
$$

for all $(w, e) \in U_{1}$.

\section{Functional DePEndence}

In this section, by exploiting Theorem 4.3, we prove Proposition 5.2 which is an important tool in the proof of Theorem 6.5 and can be viewed as a variant of the classical Implicit Function Theorem.

To this aim we extend to Banach spaces the definition of functional dependence of functions $f_{i}: U \rightarrow \mathbb{R}$, $i=1, \ldots, \kappa, U \subset E$ open, at some $x_{0} \in U$ given in Example 2.5.16 of [2].

Definition 5.1. Let $U \subset E$ be an open set and let functions $f_{i}: U \rightarrow \mathbb{R}, i=1, \ldots, \kappa$ be of class $C^{1}$ in a neighbourhood of $x_{0} \in U$. Functions $f_{1} \ldots, f_{\kappa}$ are functionally dependent at $x_{0}$ if there exist neighbourhoods $U\left(x_{0}\right), V\left(y_{0}\right)$, where $y_{0}:=\left(f_{1}\left(x_{0}\right), \ldots, f_{\kappa}\left(x_{0}\right)\right) \in \mathbb{R}^{\kappa}$ and a function $F: V\left(y_{0}\right) \rightarrow \mathbb{R}$ of class $C^{1}$ such that

1. $F\left(f_{1}(x), \ldots, f_{\kappa}(x)\right)=0$ for all $x \in U\left(x_{0}\right)$,

2. $D F\left(y_{0}\right) \neq 0$.

Functions $f_{i}: U \rightarrow \mathbb{R}, i=1, \ldots, \kappa$, are functionally independent at $x_{0}$ if $f_{i}: U \rightarrow \mathbb{R}, i=1, \ldots, \kappa$ are not functionally dependent at $x_{0}$, i.e. for any neighbourhoods $V\left(y_{0}\right), U\left(x_{0}\right)$, and for any $F: V\left(y_{0}\right) \rightarrow \mathbb{R}$ of class $C^{1}$, if $F\left(f_{1}(x), \ldots, f_{\kappa}(x)\right)=0$ for all $x \in U\left(x_{0}\right)$, then $D F\left(y_{0}\right)=0$.

Now we discuss conditions ensuring functional dependence/independence.

In the proposition below we generalize Proposition 1 of Section 8.6.3 of [29] to the case, where the argument space is a Banach space and the functional dependence is understood in the sense of Definition 5.1. Assertion 2 of the proposition below establishes a connection of CRC at $x_{0}$ and Implicit Function Theorem.

Proposition 5.2. Let $E$ be a Banach space. Let $x_{0} \in U, U \subset E, U$ - open, $f=\left[f_{1}, \ldots, f_{\kappa}\right], f_{i}: U \rightarrow \mathbb{R}, i=$ $1, \ldots, \kappa$ be $C^{1}$ functions in a neighbourhood of $x_{0}$. Assume that CRC holds at $x_{0}$ with a neighbourhood $V\left(x_{0}\right)$, i.e.

$$
\operatorname{rank}\left\{D f_{i}(x), i=1, \ldots, \kappa\right\}=\operatorname{rank}\left\{D f_{i}\left(x_{0}\right), i=1, \ldots, \kappa\right\}=k \quad \forall x \in V\left(x_{0}\right) .
$$

Let $E_{2}=\operatorname{ker} D f\left(x_{0}\right)$ and $E_{1}$ be its closed complement. Let $i_{1}, \ldots, i_{k} \subset\{1, \ldots, \kappa\}$ be such that $i_{j} \neq i_{l}$ for $j \neq l$ and $D f_{i_{1}}\left(x_{0}\right), \ldots, D f_{i_{k}}\left(x_{0}\right)$ are linearly independent.

(1) If $k=\kappa$, then functions $f_{1}, \ldots, f_{\kappa}$ are functionally independent at $x_{0}$.

(2) If $k<\kappa$, then for any $l \in\{1, \ldots, \kappa\} \backslash\left\{i_{1}, \ldots, i_{k}\right\}$ functions $f_{i_{1}} \ldots, f_{i_{k}}, f_{l}$ are functionally dependent at $x_{0}$ and there exists a function $g_{l}: \mathbb{R}^{k} \rightarrow \mathbb{R}$ of class $C^{1}$ such that for any $x$ in some neighbourhood of $x_{0}$

$$
f_{l}(x)=g_{l}\left(f_{i_{1}}(x), \ldots, f_{i_{k}}(x)\right) .
$$

Proof.

(1) The proof follows the lines of the proof of Proposition 1 of Section 8.6.3 of [29].

Let $f=\left[f_{1}, \ldots, f_{\kappa}\right]$. By Theorem 4.3, there exist diffeomorphisms of class $C^{1}, \varphi: V_{1} \rightarrow V_{2}$ and $\psi: U_{1} \rightarrow U_{2}$ such that

$$
(\varphi \circ f \circ \psi)(w, e)=(w, 0) \quad \text { for all }(w, e) \in U_{1} \subset E_{1} \oplus E_{2} \text {. }
$$


Since $\varphi, \psi$ are diffeomorphisms we have

$$
f=\varphi^{-1} \circ(\varphi \circ f \circ \psi) \circ \psi^{-1}
$$

and hence, $y_{0}:=f\left(x_{0}\right)$ is an interior point (in space $\mathbb{R}^{\kappa}$ ) of the image of a neighbourhood of $x_{0} \in E$ (note that $\left.E_{2}=\{0\}\right)$. Thus, for any function $F$, the relation

$$
F\left(f_{1}(x), \ldots, f_{\kappa}(x)\right) \equiv 0
$$

holds in a neighbourhood of $x_{0}$ only if

$$
F\left(y_{1}, \ldots, y_{\kappa}\right) \equiv 0
$$

in an neighbourhood of $y_{0}$. Hence, $D F\left(y_{0}\right)=0$.

(2) The proof follows the lines of the proof of Theorem 2.5.12 in [2].

If $\{1, \ldots, \kappa\} \backslash\left\{i_{1}, \ldots, i_{k}\right\}=\emptyset$, the assertion is automatically satisfied. Suppose that $\{1, \ldots, \kappa\} \backslash$ $\left\{i_{1}, \ldots, i_{k}\right\} \neq \emptyset$. Without loss of generality we assume that $i_{j}=j, j=1, \ldots, k$.

By Theorem 4.3, there exist open sets $U_{1} \subset \mathbb{R}^{k} \oplus E_{2}, U_{2} \subset E, V_{1} \subset \mathbb{R}^{\kappa}, V_{2} \subset \mathbb{R}^{k} \oplus E_{2}$ and diffeomorphisms of class $C^{1}, \varphi: V_{1} \rightarrow V_{2}$ and $\psi: U_{1} \rightarrow U_{2}, x_{0}=\left(x_{01}, x_{02}\right) \in U_{2} \subset U \subset E_{1} \oplus E_{2}$, i.e. $x_{01} \in E_{1}, x_{02} \in E_{2}$, $f\left(x_{0}\right) \in V_{1}$ satisfying

$$
(\varphi \circ f \circ \psi)(w, e)=(w, 0), \quad \text { where } w \in \mathbb{R}^{k}, e \in E_{2}
$$

for all $(w, e) \in U_{1}$. Note that the diffeomorphism $\psi$ is the same as in Theorem 2.5. Hence,

$$
\bar{f}(w, e):=(f \circ \psi)(w, e)=\varphi^{-1}(w, 0)=(w, \eta(w, e)),
$$

where $\eta: \mathbb{R}^{k} \times E_{2} \rightarrow E_{2}$ is the same as in Theorem 2.5. Thus, $\bar{f}$ does not depend on $e \in E_{2}$.

Let $x \in U_{2}$ and denote

$$
y_{i}=f_{i}(x), \quad i=1, \ldots, \kappa .
$$

There exists $u=(w, e) \in U_{1} \in \mathbb{R}^{k} \oplus E_{2}$ such that $x=\psi(u)$. Hence,

$$
y_{j}=f_{j}(\psi(w, e))=w_{j}, \quad j=1, \ldots, k .
$$

For $l \in\{k+1, \ldots, \kappa\}$ we have

$$
y_{l}=f_{l}(x)=f_{l}(\psi(w, e))=\bar{f}_{l}(w, e)=\bar{f}_{l}\left(y_{1}, \ldots, y_{k}, e\right) .
$$

In consequence, by (5.2), $y_{l}=\bar{f}_{l}\left(y_{1}, \ldots, y_{k}\right), l \in\{k+1, \ldots, \kappa\}$. Hence, for any $x \in U_{2}, f_{l}(x)=$ $\bar{f}_{l}\left(f_{1}(x), \ldots, f_{k}(x)\right), l \in\{k+1, \ldots, \kappa\}$.

The following example illustrates functional independence of functions at $x_{0}$ under CRC.

Example 5.3. Let $\ell_{2}$ be the Hilbert space of square summable series. Let $f_{1}, f_{2}: \ell_{2} \rightarrow \mathbb{R}$ be given as $f_{1}(x)=x_{1}$, $f_{2}(x)=x_{2}$, where $x=\left(x_{1}, x_{2}, \ldots\right) \in \ell_{2}$. We will show that $f_{1}, f_{2}$ are functionally independent at $x_{0}=0 \in \ell_{2}$. Suppose, by contrary, that $f_{1}, f_{2}$ are functionally dependent, i.e there exists a function $F: \mathbb{R}^{2} \rightarrow \mathbb{R}$ of class $C^{1}$ such that $F\left(f_{1}(x), f_{2}(x)\right)=0$ for all $x$ in some neighbourhood of $0 \in \ell_{2}$ and $D F \neq 0$ in some neighbourhood 
of $\left(f_{1}(0), f_{2}(0)\right)=(0,0) \in \mathbb{R}^{2}$. Indeed, by Implicit Function Theorem (see e.g. [2], Thm. 2.5.7), there exists $g: \mathbb{R} \rightarrow \mathbb{R}$ such that $f_{2}(x)=g\left(f_{1}(x)\right)$ for all $x$ in some neighbourhood of $x_{0}$ and

$$
D f_{2}(x)=\left[\begin{array}{c}
\frac{d g}{d f_{1}}\left(f_{1}(x)\right) \cdot \frac{\partial f_{1}}{\partial x_{1}} \\
\frac{d g}{d f_{1}}\left(f_{1}(x)\right) \cdot \frac{\partial f_{1}}{d x_{2}} \\
0 \\
\vdots
\end{array}\right]^{T}=\frac{d g}{d f_{1}}\left(f_{1}(x)\right) D f_{1}(x)
$$

i.e. $D f_{1}(x), D f_{2}(x)$ are linearly dependent for all $x$ in some neighbourhood of $0 \in \ell_{2}$, which is not true.

\section{TANGENT AND LINEARIZED CONES}

In the present section we prove our main result, namely the Abadie condition in Banach space for the set $\mathcal{F}$ given by (1.1) under RCRCQ. The finite-dimensional case has been proved by Minchenko and Stakhovski in [23].

Let $E$ be a Banach space. Let $C$ be a subset of $E$ and $x_{0} \in \operatorname{cl} C$. We use the classical definition of tangent cone of $C$ at $x_{0}$,

$$
\begin{aligned}
T_{C}\left(x_{0}\right):=\{ & d \in E \mid \exists \varepsilon>0 \\
& \exists \text { a vector function } o(t) \text { such that }\|o(t)\| t^{-1} \rightarrow 0, \text { as } t \downarrow 0 \\
& \text { and } \left.x_{0}+t d+o(t) \in C \forall 0 \leq t \leq \varepsilon\right\} .
\end{aligned}
$$

For the set $\mathcal{F}$ given by $(1.1)$ and $x_{0} \in \mathcal{F}$, the linearized cone is given as

$$
\Gamma_{\mathcal{F}}\left(x_{0}\right):=\left\{d \in E \mid\left\langle D h_{i}\left(x_{0}\right) \mid d\right\rangle \leq 0, i \in I\left(x_{0}\right),\left\langle D h_{i}\left(x_{0}\right) \mid d\right\rangle=0, i \in I_{0}\right\},
$$

where $I\left(x_{0}\right):=\left\{i \in I \mid h_{i}\left(x_{0}\right)=0\right\}$ is the active index set of $\mathcal{F}$ at $x_{0}$.

Definition 6.1 (Relaxed Constant Rank Constraint Qualification). The relaxed constant rank constraint qualification (RCRCQ) holds for set $\mathcal{F}$, given by (1.1) at $\bar{x} \in \mathcal{F}$, if there exists a neighbourhood $U(\bar{x})$ of $\bar{x}$ such that, for any index set $J, I_{0} \subset J \subset I_{0} \cup I(\bar{x})$, for every $x \in U(\bar{x})$, the system of vectors $\left\{D h_{i}(x), i \in J\right\}$ has constant rank. Precisely, for any $J, I_{0} \subset J \subset I_{0} \cup I(\bar{x})$,

$$
\operatorname{rank}\left(D h_{i}(x), i \in J\right)=\operatorname{rank}\left(D h_{i}(\bar{x}), i \in J\right) \text { for all } x \in U(\bar{x}) .
$$

Remark 6.2. Note that RCRCQ holds for $\mathcal{F}$ at $x_{0} \in \mathcal{F}$ if and only if for any index set $J, I_{0} \subset J \subset I_{0} \cup I\left(x_{0}\right)$, CRC holds at $x_{0}$ for functions $h_{i}, i \in J$.

In Theorem 6.5 we will use Ljusternik theorem (see [16], Sect. 0.2.4).

Theorem 6.3. (Ljusternik Theorem) Let $X$ and $Y$ be Banach spaces, let $U$ be a neighborhood of a point $x_{0} \in X$, and let $F: U \rightarrow Y$ be a Fréchet differentiable mapping. Assume that $F$ is regular at $x_{0}$, i.e., that $\operatorname{Im} D F\left(x_{0}\right)=Y$, and that its derivative is continuous at this point (in the uniform operator topology of the space $\mathcal{L}(X, Y))$. Then the tangent space $T_{M}\left(x_{0}\right)$ to the set

$$
M=\left\{x \in U \mid F(x)=F\left(x_{0}\right)\right\}
$$

at the point $x_{0}$ coincides with the kernel of the operator $D F\left(x_{0}\right)$,

$$
T_{M}\left(x_{0}\right)=\operatorname{Ker} D F\left(x_{0}\right) .
$$


Moreover, if the assumptions of the theorem are satisfied, then there exist a neighborhood $U^{\prime} \subset U$ of the point $x_{0}$, a number $K>0$, and a mapping $\xi \rightarrow x(\xi)$ of the set $U^{\prime}$ into $X$ such that

$$
\begin{aligned}
& F(\xi+x(\xi))=F\left(x_{0}\right), \\
& \|x(\xi)\| \leq K\left\|F(\xi)-F\left(x_{0}\right)\right\|
\end{aligned}
$$

for all $\xi \in U^{\prime}$.

We start with the following technical lemma (see also [23] for finite-dimensional case).

Lemma 6.4. Let $x_{0} \in \mathcal{F}$, where $\mathcal{F}$ is given by (1.1) and $d \in \Gamma_{\mathcal{F}}\left(x_{0}\right)$. For any vector function $r:(0,1) \rightarrow E$ such that $\|r(t)\| t^{-1} \rightarrow 0$, as $t \downarrow 0$, there exists a number $\varepsilon_{0}>0$ such that

$$
h_{i}\left(x_{0}+t d+r(t)\right)<0 \text { for all } i \in I \backslash I\left(x_{0}, d\right) \text { and for all } t \in\left(0, \varepsilon_{0}\right) \text {, }
$$

where $I\left(x_{0}, d\right):=\left\{i \in I\left(x_{0}\right) \mid\left\langle D h_{i}\left(x_{0}\right), d\right\rangle=0\right\}$.

Proof. Let $d \in \Gamma_{\mathcal{F}}\left(x_{0}\right)$. If $i \in I \backslash I\left(x_{0}\right)$, then $h_{i}\left(x_{0}\right)<0$ and, therefore,

$$
\begin{aligned}
h_{i}\left(x_{0}\right. & +t d+r(t))=h_{i}\left(x_{0}\right)+\left\langle D h_{i}\left(x_{0}+\theta(t d+r(t))\right), t d+r(t)\right\rangle \\
& =h_{i}\left(x_{0}\right)+t\left\langle D h_{i}\left(x_{0}+\theta(t d+r(t)), d\right\rangle+t\left\langle D h_{i}\left(x_{0}+\theta(t d+r(t))\right), \frac{r(t)}{t}\right\rangle<0,\right.
\end{aligned}
$$

where $0 \leq \theta \leq 1$ for all sufficiently small $t>0$.

If $i \in I\left(x_{0}\right) \backslash I\left(x_{0}, d\right)$, then

$$
h_{i}\left(x_{0}+t d+r(t)\right)=h_{i}\left(x_{0}\right)+t\left\langle D h_{i}\left(x_{0}\right), d\right\rangle+o_{i}(t)=t\left\langle D h_{i}\left(x_{0}\right), d\right\rangle+o_{i}(t),
$$

where

$$
\begin{aligned}
o_{i}(t) & :=t\left\langle D h_{i}\left(x_{0}+\theta(t d+r(t)), d\right\rangle+t\left\langle D h_{i}\left(x_{0}+\theta(t d+r(t))\right), \frac{r(t)}{t}\right\rangle\right. \\
& -t\left\langle D h_{i}\left(x_{0}\right), d\right\rangle .
\end{aligned}
$$

In this case $h_{i}\left(x_{0}+t d+r(t)\right)<0$ for sufficiently small $t>0$ since

$$
\left\langle D h_{i}\left(x_{0}\right), d\right\rangle<0 \text { and } o_{i}(t) t^{-1} \rightarrow 0 \text {. }
$$

Consequently, $h_{i}\left(x_{0}+t d+r(t)\right)<0$, for all $i \in I \backslash I\left(x_{0}, d\right)$ and for all $t \in\left(0, \varepsilon_{0}\right)$, which proves (6.1).

Let us note that Lemma 6.4 is valid also in the case $I\left(x_{0}, d\right)=\emptyset$. Now we are ready to prove our main result.

Theorem 6.5. Let $E$ be a Banach space and $\mathcal{F} \subset E$ be given as in (1.1). Assume that $R C R C Q$ holds for $\mathcal{F}$ at $x_{0} \in \mathcal{F}$. Then Abadie condition holds, i.e. $\Gamma_{\mathcal{F}}\left(x_{0}\right)=T_{\mathcal{F}}\left(x_{0}\right)$.

Moreover, for each $d \in T_{\mathcal{F}}\left(x_{0}\right)$ there is a vector function $r:(0,1) \rightarrow E,\|r(t)\| / t \rightarrow 0$ when $t \downarrow 0$, such that for all $t$ sufficiently small

$$
\begin{aligned}
& h_{i}\left(x_{0}+t d+r(t)\right)=0, \quad i \in J(d), \\
& h_{\ell}\left(x_{0}+t d+r(t)\right) \leq 0, \quad \ell \in I \backslash J(d), \quad J(d):=I_{0} \cup I\left(x_{0}, d\right) .
\end{aligned}
$$


Additionally, whenever $J(d) \neq \emptyset, d \in \operatorname{ker} \operatorname{Dh}\left(x_{0}\right)$, where $h:=\left[h_{i_{j}}\right], j=1, \ldots, k$,

$$
\operatorname{rank}\left\{D h_{i}\left(x_{0}+t d+r(t)\right), i \in J(d)\right\}=\operatorname{rank}\left\{D h_{i}\left(x_{0}\right), i \in J(d)\right\}=k
$$

for all $t$ sufficiently small.

Proof. The inclusion $T_{\mathcal{F}}\left(x_{0}\right) \subset \Gamma_{\mathcal{F}}\left(x_{0}\right)$ is immediate. To see the converse, take any $d \in \Gamma_{\mathcal{F}}\left(x_{0}\right)$. We start by considering the case $J:=J(d) \neq \emptyset$. By RCRCQ of $\mathcal{F}$ at $x_{0}$, we have

$$
\operatorname{rank}\left\{D h_{i}\left(x_{0}+t d+r\right), i \in J\right\}=\operatorname{rank}\left\{D h_{i}\left(x_{0}\right), i \in J\right\}=k,
$$

for $(t, r)$ in some neighbourhood of $(0,0) \in \mathbb{R} \times E$. By Lemma 2.4, there exist indices $i_{1}, \ldots, i_{k}$, such that $D h_{i_{1}}\left(x_{0}+t d+r\right), \ldots, D h_{i_{k}}\left(x_{0}+t d+r\right)$ are linearly independent for $(t, r)$ in some neighbourhood of $(0,0)$. Without loss of generality, we can assume that $i_{j}=j, j=1, \ldots, k$.

If $k=|J|$, i.e. $J \backslash\{1, \ldots, k\}=\emptyset$, then, by applying Ljusternik Theorem 6.3 to

$$
M:=\left\{x \in E \mid h_{i}(x)=h_{i}\left(x_{0}\right)=0, i \in J=\{1,2, \ldots, k\}\right\}
$$

the conclusion holds.

If $k<|J|$ then, by (2) of Proposition 5.2, applied to $h_{i}, i \in 1 \ldots, k$, there exist functions $g_{l}, l \in J \backslash\{1, \ldots, k\}$ of class $C^{1}$, such that

$$
h_{l}\left(x_{0}+t d+r\right)=g_{l}\left(h_{1}\left(x_{0}+t d+r\right), \ldots, h_{k}\left(x_{0}+t d+r\right)\right),
$$

for $(t, r)$ in some neighbourhood of $(0,0)$.

Consider the system

$$
h_{i}\left(x_{0}+t d+r\right)=0, \quad i \in J
$$

with respect to variables $t, r$. Let us note that system (6.4) is satisfied for $(t, r)=(0,0)$.

Obviously, in some neighbourhood of $(0,0)$, system (6.4) is equivalent to

$$
\left\{\begin{array}{c}
h_{1}\left(x_{0}+t d+r\right)=0 \\
\ldots \\
h_{k}\left(x_{0}+t d+r\right)=0
\end{array}\right.
$$

with additional condition

$$
h_{l}\left(x_{0}+t d+r\right)=g_{l}\left(h_{1}\left(x_{0}+t d+r\right), \ldots, h_{k}\left(x_{0}+t d+r\right)\right)=0, l \in J \backslash\{1, \ldots, k\} .
$$

Note that $g_{l}\left(h_{1}\left(x_{0}\right), \ldots, h_{k}\left(x_{0}\right)\right)=0, l \in J \backslash\{1, \ldots, k\}$ and therefore $g_{l}(0, \ldots, 0)=0, l \in J \backslash\{1, \ldots, k\}$.

We have

$$
\left\langle D h_{i}\left(x_{0}\right), d\right\rangle=0, \quad i \in J=I_{0} \cup I\left(x_{0}, d\right) .
$$

Hence, $d \in \operatorname{ker} D h\left(x_{0}\right)$, where $h(x)=\left[h_{1}(x) \ldots, h_{k}(x)\right]$. By applying Ljusternik Theorem 6.3 with $F=h$ at $x_{0}$, we obtain that $d \in T_{M}\left(x_{0}\right)$, where

$$
M:=\{x \in E \mid h(x)=0\} .
$$


This means that there exist $\varepsilon>0$ and a function $r:[0, \varepsilon) \rightarrow E,\|r(t)\| t^{-1} \rightarrow 0, t \downarrow 0$, such that

$$
\left\{\begin{array}{c}
h_{1}\left(x_{0}+t d+r(t)\right)=0 \\
\ldots \\
h_{k}\left(x_{0}+t d+r(t)\right)=0 .
\end{array}\right.
$$

By (6.6), $h_{i}\left(x_{0}+t d+r(t)\right)=0, i \in J$ for $t \in[0, \varepsilon]$. By Lemma 6.4, there exists $\varepsilon_{0}>0$ such that

$$
x_{0}+t d+r(t) \in \mathcal{F} \quad t \in\left[0, \min \left\{\varepsilon_{0}, \varepsilon\right\}\right] .
$$

Thus, $d \in T_{\mathcal{F}}\left(x_{0}\right)$.

Now, let us consider the case $J=\emptyset$ (i.e. the case when both $I_{0}=\emptyset$ and $\left.I\left(x_{0}, d\right)=\emptyset\right)$. Then, by Lemma 5.1, for any vector function $r:(0,1) \rightarrow E,\|r(t)\| / t \rightarrow 0$ when $t \downarrow 0$ there exists $\varepsilon>0$ such that

$$
x_{0}+t d+r(t) \in \mathcal{F} \quad t \in[0, \varepsilon],
$$

i.e., $d \in T_{\mathcal{F}}\left(x_{0}\right)$.

The following corollary refers to the special case, where there is no inequality constraints in the definition of the set $\mathcal{F}$.

Corollary 6.6. Suppose that $I=\emptyset$, i.e. there is no inequalities in the representation (1.1) of the set $\mathcal{F}$ i.e.

$$
\mathcal{F}=\left\{x \in E \mid h_{i}(x)=0 \quad i=1, \ldots, n\right\}
$$

and $C R C$ holds at $x_{0} \in \mathcal{F}$, i.e. there exists a neigbourhood $U\left(x_{0}\right)$ s.t.

$$
\operatorname{rank}\left\{D h_{i}\left(x_{0}\right), i=1,2, \ldots, n\right\}=\operatorname{rank}\left\{D h_{i}(x), i=1,2, \ldots, n\right\}=k
$$

for all $x \in U\left(x_{0}\right)$. Then

$$
T_{\mathcal{F}}\left(x_{0}\right)=\left\{d:\left\langle D h_{i}\left(x_{0}\right), d\right\rangle=0, i=1,2, \ldots, n\right\} .
$$

Moreover, if $I_{k}=\left\{i_{1}, i_{2}, \ldots, i_{k}\right\}$ is such that $D h_{i_{j}}\left(x_{0}\right), i_{j} \in I_{k}$ are linearly independent, then

$$
T_{\mathcal{F}}\left(x_{0}\right)=\operatorname{ker}\left[\begin{array}{c}
D h_{i_{1}}\left(x_{0}\right) \\
\vdots \\
D h_{i_{k}}\left(x_{0}\right)
\end{array}\right] .
$$

Proof. By assumption, for any $\ell \notin I_{k}$

$$
D h_{\ell}\left(x_{0}\right)=\sum_{i \in I_{k}} \lambda_{i}^{\ell} D h_{i}\left(x_{0}\right)
$$

This shows that $T_{\mathcal{F}}\left(x_{0}\right)$ does not depend upon the choice of the set $I_{k}$.

\section{Functional dependence/Independence without CRC}

In our main theorem (Thm. 6.5) we used constant rank condition (and RCRCQ) to be able to apply Proposition 5.2, where we used the concept of functional dependence/independence according to Definition 5.1. 
In this additional section we investigate functional dependence/independence with respect to Definition 5.1 without CRC. Moreover, in Subsection 7.1 we review other most common concepts of functional dependence/independence (Defs. 7.5, 7.9, 7.13).

Proposition 7.1. Let $E$ be a Banach space. Let $x_{0} \in U, U \subset E$ open and $f_{1}, \ldots, f_{\kappa}: U \rightarrow \mathbb{R}$. Suppose that in every neighbourhood $U\left(x_{0}\right)$ there exists $x \in U\left(x_{0}\right)$ such that $D f_{1}(x), \ldots, D f_{n}(x)$ are linearly independent. Then functions $f_{1}, \ldots, f_{\kappa}$ are functionally independent at $x_{0}$.

Proof. Let $F: \quad V \rightarrow \mathbb{R}$ be a function of class $C^{1}$ defined on a neighbourhood $V\left(y_{0}\right)$ such that $F\left(f_{1}(x), \ldots, f_{\kappa}(x)\right)=0$ for any $x$ in some neighbourhood $U\left(x_{0}\right)$.

We show that it must be $D F\left(y_{0}\right)=0$, where $y_{0}=\left(f_{1}\left(x_{0}\right), \ldots, f_{\kappa}\left(x_{0}\right)\right)$. By assumption, let $U\left(x_{0}\right)$ be a neighbourhood of $x_{0}$ and $x^{\prime} \in U\left(x_{0}\right)$ be such that $D f_{1}\left(x^{\prime}\right), \ldots, D f_{\kappa}\left(x^{\prime}\right)$ are linearly independent.

There exists a neighbourhood $U\left(x^{\prime}\right) \subset U\left(x_{0}\right)$ and $D f_{1}\left(z^{\prime}\right), \ldots, D f_{\kappa}\left(z^{\prime}\right)$ are linearly independent for all $z \in$ $U\left(x^{\prime}\right)$. By (1) of Proposition 5.2, it must be $D F\left(f\left(x^{\prime}\right)\right)=0$. By smoothness of function $F$ and $f$, the latter equality implies $D F\left(f\left(x_{0}\right)\right)=0$.

Proposition 7.2. (Local stability of functional dependence) Let $E$ be a Banach space. If $f_{1}, \ldots, f_{\kappa}: U \rightarrow \mathbb{R}$, $U \subset E$ open, are functionally dependent at $x_{0} \in U$, then there exists a neighbourhood $U\left(x_{0}\right)$ such that $f_{1}, \ldots, f_{\kappa}$ are functionally dependent at any $x \in U\left(x_{0}\right)$.

Proof. Let $f=\left[f_{1}, \ldots, f_{\kappa}\right]$. Assume that $f_{1}, \ldots, f_{\kappa}$ are functionally dependent at $x_{0}$, i.e. there exist neighbourhoods $U\left(x_{0}\right), V\left(y_{0}\right), y_{0}=f\left(x_{0}\right)$, and a function $F: V\left(y_{0}\right) \rightarrow \mathbb{R}$ such that $D F\left(y_{0}\right) \neq 0$ and $F\left(f_{1}(x), \ldots, f_{\kappa}(x)\right)=$ 0 for all $x \in U\left(x_{0}\right)$.

Since $D F\left(y_{0}\right) \neq 0$ and $F$ is of class $C^{1}$ there exists a neighbourhood $V_{1}\left(y_{0}\right)$ such that $D F(y) \neq 0$ for all $y \in V_{1}\left(y_{0}\right)$. Let $x^{\prime} \in U\left(x_{0}\right) \cap f^{-1}\left(V_{1}\left(y_{0}\right)\right), U\left(x^{\prime}\right):=U\left(x_{0}\right) \cap f^{-1}\left(V_{1}\left(y_{0}\right)\right)$ and $y^{\prime}:=f\left(x^{\prime}\right)$. Then function $F$ : $V_{1}\left(y_{0}\right) \cap V\left(y_{0}\right) \rightarrow \mathbb{R}$ satisfies $D F\left(y^{\prime}\right) \neq 0$ and $F\left(f_{1}(x), \ldots, f_{\kappa}(x)\right)=0$ for all $x \in U\left(x^{\prime}\right)$. Hence $f_{1}, \ldots, f_{\kappa}$ are functionally dependent at any $x^{\prime} \in U\left(x_{0}\right) \cap f^{-1}\left(V_{1}\left(y_{0}\right)\right)$.

The fact below relates functional dependence with linear dependence of gradients.

Fact 7.3. Let $E$ be a Banach space. Let $x_{0} \in U, U \subset E$ open, and $f_{1}, \ldots, f_{\kappa}: U \rightarrow \mathbb{R}$. Suppose that $f_{1}, \ldots, f_{\kappa}$ are functionally dependent at $x_{0}$. Then there exists a neighbourhood $U\left(x_{0}\right)$ such that $D f_{1}(x), \ldots, D f_{\kappa}(x)$, $x \in U\left(x_{0}\right)$, are linearly dependent.

Proof. The proof follows immediately from Lemma 2.4, (1) of Proposition 5.2 and Proposition 7.2.

The following proposition provides sufficient conditions for functional independence.

Proposition 7.4. Let $x_{0} \in U, U \subset E$ open, $f_{1}, \ldots, f_{\kappa}: U \rightarrow \mathbb{R}$. If, for any neighbourhood $U\left(x_{0}\right)$, int $f\left(U\left(x_{0}\right)\right) \neq \emptyset$, then $f_{1}, \ldots, f_{\kappa}$ are functionally independent at $x_{0}$.

Proof. Let $E$ be a Banach space. Let $U\left(x_{0}\right)$ be a neighbourhood of $x_{0}$ and $V\left(y_{0}\right)$ be a neighbourhood of $y_{0}=f\left(x_{0}\right)$ and $F: V \rightarrow \mathbb{R}$ be of class $C^{1}$ such that $F\left(f_{1}(x), \ldots, f_{\kappa}(x)\right)=0$ for all $x \in U\left(x_{0}\right)$.

By the continuity of $f$, for any $m \in \mathbb{N}$ there exists $U_{m}^{\prime}\left(x_{0}\right)$ such that $f\left(U_{m}^{\prime}\left(x_{0}\right)\right) \subset B\left(y_{0}, \frac{1}{m}\right)$. Let $U_{m}^{\prime \prime}\left(x_{0}\right)=$ $U\left(x_{0}\right) \cap U_{m}^{\prime}\left(x_{0}\right)$. Then, by assumption, $\operatorname{int}\left(f\left(U_{m}^{\prime \prime}\left(x_{0}\right)\right)\right) \neq \emptyset$ and, moreover, $A_{m}:=\left[\operatorname{int} f\left(U_{m}^{\prime \prime}\left(x_{0}\right)\right)\right] \cap B\left(y_{0}, \frac{1}{m}\right)=$ $\operatorname{int} f\left(U_{m}^{\prime \prime}\left(x_{0}\right)\right)$ is a nonempty open set. Since $F(y)=0$ for all $y \in A_{m}$ we have $D F(y)=0$ for all $y \in A_{m}$.

Since $F: V \rightarrow \mathbb{R}$ is of class $C^{1}$, there exists a sequence $y_{m} \rightarrow y_{0}, y_{m} \in A_{n}$, such that $D F\left(y_{m}\right)=0$. By the smoothness of $F$, it must be $D F\left(y_{0}\right)=0$. In consequence, functions $f_{1}, \ldots, f_{\kappa}$ are functionally independent at $x_{0}$.

\subsection{Functional dependence/independence, other definitions}

Here we compare the concept of functional dependence given in Definition 5.1 with other concepts of functional dependence appearing in the literature. 
Let us note that in the proof of Theorem 6.5 and Proposition 7.4 we use the concept of functional dependence as defined in Definition 5.1. In general, without condition 2. of Definition 5.1 we are not able to deduce formula (6.3).

The definition of functional dependence at $x_{0}$ given in Chapter II.7 of [11] can be rewritten in Banach spaces as follows.

Definition 7.5. Let $E$ be a Banach space. Let $U \subset E$ be an open set and let functions $f_{i}: U \rightarrow \mathbb{R}, i=1, \ldots, \kappa$ be of class $C^{1}$ in a neighbourhood of $x \in U$. Functions $f_{1} \ldots, f_{\kappa}$ are said to be functionally dependent at $x_{0}$ if there exists a neighbourhood $U\left(x_{0}\right)$ and a neighbourhood $V\left(y_{0}\right)$, where $y_{0}:=\left(f_{1}\left(x_{0}\right), \ldots, f_{\kappa}\left(x_{0}\right)\right) \in \mathbb{R}^{\kappa}$ and a function $F: V \rightarrow \mathbb{R}$ of class $C^{1}$ such that

1. $F\left(f_{1}(x), \ldots, f_{\kappa}(x)\right)=0$ for all $x \in U\left(x_{0}\right)$,

2. $F \not \equiv 0$ on $V\left(y_{0}\right)$.

Definition 7.5 for continuous functions $f_{1} \ldots, f_{\kappa}, F$ in finite-dimensional settings was given in Paragraph 8.6.3 of [29].

Remark 7.6. Clearly, if $f_{1}, \ldots, f_{\kappa}$ are functionally dependent at $x_{0}$ in the sense of Definition 5.1 , then $f_{1}, \ldots, f_{\kappa}$ are functionally dependent at $x_{0}$ in the sense of Definition 7.5.

The example below illustrates the difference between of definitions of functional dependence given in Definition 5.1 and Definition 7.5. Let us note that the functions $f_{1}, f_{2}$ from the example below do not satisfy the CRC condition at $x_{0}=(0,0)$.

Example 7.7. Let $f_{1}, f_{2}: \mathbb{R}^{2} \rightarrow \mathbb{R}$ be defined as $f_{1}\left(x_{1}, x_{2}\right)=x_{1}^{2}, f_{2}\left(x_{1}, x_{2}\right)=x_{2}^{2}$ and $x_{0}=(0,0)$. We will show that $f_{1}, f_{2}$ are functionally dependent at $x_{0}$ in the sense of Definition 7.5 and are functionally independent at $x_{0}$ in the sense of Definition 5.1.

Let $F: \mathbb{R}^{2} \rightarrow \mathbb{R}$ be defined as follows

$$
F\left(y_{1}, y_{2}\right):= \begin{cases}0 & \text { if } y_{1} \geq 0 \wedge y_{2} \geq 0 \\ y_{1}^{2} & \text { if } y_{1}<0 \wedge y_{2} \geq 0 \\ y_{2}^{2} & \text { if } y_{1} \geq 0 \wedge y_{2}<0 \\ y_{1}^{2}+y_{2}^{2} & \text { if } y_{1}<0 \wedge y_{2}<0\end{cases}
$$

Then $F$ is of class $C^{1}$ and

$$
F\left(f_{1}\left(x_{1}, x_{2}\right), f_{2}\left(x_{1}, x_{2}\right)\right)=F\left(x_{1}^{2}, x_{2}^{2}\right)=0
$$

for all $\left(x_{1}, x_{2}\right) \in \mathbb{R}^{2}$. Moreover, in any neighbourhood of $y_{0}:=\left(f_{1}\left(x_{0}\right), f_{2}\left(x_{0}\right)\right)=(0,0)$ there exists $y=\left(y_{1}, y_{2}\right)$ such that $y_{1}<0$ or $y_{2}<0$, i.e. $F(y) \neq 0$. Hence, $f_{1}, f_{2}$ are functionally dependent at $x_{0}$ in the sense of Definition 7.5

Now we show that $f_{1}, f_{2}$ are functionally independent at $x_{0}$ in the sense of Definition 5.1. By contrary, suppose, that $f_{1}, f_{2}$ are functionally dependent at $x_{0}$ in the sense of Definition 5.1. Then there exists a function $F: \mathbb{R}^{2} \rightarrow \mathbb{R}$ of class $C^{1}$ such that $D F\left(y_{0}\right) \neq 0$ at $y_{0}:=\left(f_{1}\left(x_{0}\right), f_{2}\left(x_{0}\right)\right)=(0,0)$ and $F\left(x_{1}^{2}, x_{2}^{2}\right)=0$ for all $\left(x_{1}, x_{2}\right)$ in some neighbourhood of $x_{0}$.

Let $U\left(x_{0}\right)$ be any neighbourhood of $x_{0}$ and $V\left(y_{0}\right)$ be any neighbourhood of $y_{0}$. Let $U^{\prime}\left(x_{0}\right)=\left\{\left(x_{1}, x_{2}\right) \in \mathbb{R}^{2} \mid\right.$ $\left.\left(\operatorname{sgn}\left(x_{1}\right) x_{1}^{2}, \operatorname{sgn}\left(x_{2}\right) x_{2}^{2}\right) \in U\left(x_{0}\right)\right\}$, where sgn is the signum function. Let us take any $y^{\prime} \in V\left(y_{0}\right) \cap U^{\prime}\left(x_{0}\right)$ such that $y^{\prime}=\left(y_{1}^{\prime}, y_{2}^{\prime}\right)$, where $y_{1}^{\prime}>0$ and $y_{2}^{\prime}>0$. Let $V\left(y^{\prime}\right)=V\left(y_{0}\right) \cap U^{\prime}\left(x_{0}\right) \cap \mathbb{R}_{++}^{2}$, where $R_{++}^{2}:=\left\{\left(x_{1}, x_{2}\right) \mid x_{1}>\right.$ $\left.0 \wedge x_{2}>0\right\}$. Let us note that $y_{0} \in \operatorname{cl}\left(V\left(y^{\prime}\right)\right)$. Then for all $y=\left(y_{1}, y_{2}\right) \in V\left(y^{\prime}\right)$ we have $\left(\sqrt{y_{1}}, \sqrt{y_{2}}\right) \in U^{\prime}\left(x_{0}\right)$ and, moreover, $F(y)=F\left({\sqrt{y_{1}}}^{2},{\sqrt{y_{2}}}^{2}\right)=0$. Thus, $D F(y)=0$ for all $y \in V\left(y^{\prime}\right) \subset V\left(y_{0}\right)$, which is a contradiction with the assumption $\operatorname{DF}\left(y_{0}\right) \neq 0$. 
Definition 7.8. Let $\Omega \subset E$ be a nonempty set. Subset $A \subset \Omega$ is nowhere dense in $\Omega$ if for all $U \subset \Omega, U$ open in $\Omega, U \neq \emptyset$ there exists $V \subset U, V$ open in $\Omega, V \neq \emptyset$ such that $A \cap V=\emptyset$, i.e. $A \subset \Omega \backslash V$.

The definition of functional dependence on a set $\Omega$ given in Chapter 1 of [24] is formulated in Banach spaces for $C^{\infty}$ functions. Here we reformulate it in Banach space settings for $C^{1}$ functions in the following way.

Definition 7.9. Let $E$ be a Banach space. Let $\Omega \subset E$ be an open set and let functions $f_{i}: \Omega \rightarrow \mathbb{R}, i=1, \ldots, \kappa$ be of class $C^{1}$ in a neighbourhood of $x_{0} \in \Omega$. Functions $f_{1} \ldots, f_{\kappa}$ are said to be functionally dependent at $x_{0}$ if there exist a neighbourhood $U\left(x_{0}\right) \subset \Omega$ and a neighbourhood $V\left(y_{0}\right), y_{0}:=\left(f_{1}\left(x_{0}\right), \ldots, f_{\kappa}\left(x_{0}\right)\right) \in \mathbb{R}^{\kappa}$, and a function $F: V\left(y_{0}\right) \rightarrow \mathbb{R}$ of class $C^{1}$ such that

1. $F\left(f_{1}(x), \ldots, f_{\kappa}(x)\right)=0$ for all $x \in U\left(x_{0}\right)$,

2. $F^{-1}(0)$ is nowhere dense in $V\left(y_{0}\right)$.

The following example shows that the functional dependence in the sense of Definition 7.9 does not imply functional dependence in the sense of Definition 5.1.

Example 7.10. Let $f_{1}(t)=t^{3}, f_{2}(t)=t^{2}, t \in \mathbb{R}$. Then functions $f_{1}, f_{2}$ are functionally dependent at $t=0$ in the sense of Definition 7.9 , since by taking $F(x, y)=x^{3}-y^{2}$ we get:

1. $F\left(f_{1}(t), f_{2}(t)\right)=0$ for all $t \in \mathbb{R}$

2. $F^{-1}(0)$ is nowhere dense in any neighbourhood of $(0,0)$.

On the other hand for any neighbourhood of $U(0)$ and $V((0,0))$ and for any function $F: V((0,0)) \rightarrow \mathbb{R}$ of class $C^{1}$ if $F\left(f_{1}(t), f_{2}(t)\right)=0$ for all $t \in U(0)$, then

$$
D F(0,0)=\frac{\partial F\left(f_{1}(0), f_{2}(0)\right)}{\partial f_{1}} \frac{d f_{1}(0)}{d t}+\frac{\partial F\left(f_{1}(0), f_{2}(0)\right)}{\partial f_{2}} \frac{d f_{2}(0)}{d t}=0 .
$$

Therefore functions $f_{1}, f_{2}$ are functionally independent at $t=0$ in the sense of Definition 5.1.

Proposition 7.11. Let $E$ be a Banach space. If functions $f_{i}: \Omega \rightarrow \mathbb{R}, i=1, \ldots, \kappa$ of class $C^{1}$ are functionally dependent at $x_{0} \in \Omega$ in the sense of Definition 7.9 then they are functionally dependent in the sense of Definition 7.5.

Proof. Assume that that $f_{1}, \ldots, f_{\kappa}$ are functionally dependent at $x_{0} \in \Omega$ in the sense of Definition 7.9. Then there are neighbourhood $U\left(x_{0}\right)$ and a neighbourhood $V\left(y_{0}\right), \quad V\left(y_{0}\right) \subset f\left(U\left(x_{0}\right)\right)$, where $y_{0}:=$ $\left(f_{1}\left(x_{0}\right), \ldots, f_{\kappa}\left(x_{0}\right)\right) \in \mathbb{R}^{\kappa}$ and a function $F: V \rightarrow \mathbb{R}$ of class $C^{1}$ such that

1. $F\left(f_{1}(x), \ldots, f_{\kappa}(x)\right)=0$ for all $x \in U\left(x_{0}\right)$,

2. $F^{-1}(0)$ is nowhere dense in $V\left(y_{0}\right)$.

According to the definition of nowhere dense set, for every nonempty open set $U \subset V\left(y_{0}\right)$, there exists an open set a nonempty set $V \subset U$, such that $V \cap F^{-1}(0)=\emptyset$, i.e. $F(x) \neq 0$ for every $x \in V$. In conclusion, $F$ satisfies condition 2. of Definition 7.5.

Proposition 7.12. Let $E$ be a Banach space. If functions $f_{i}: \Omega \rightarrow \mathbb{R}, i=1, \ldots, \kappa$ of class $C^{1}$ are functionally dependent at $x_{0} \in \Omega$ in the sense of Definition 7.9, then there exists a neighbourhood $U\left(x_{0}\right)$ such that $D f_{1}(x), \ldots, D f_{\kappa}(x), x \in U\left(x_{0}\right)$ are linearly dependent.

Proof. Suppose, by contrary that for any neighbourhood $U\left(x_{0}\right)$ there exists $x^{\prime} \in U\left(x_{0}\right)$ such that $D f_{1}\left(x^{\prime}\right), \ldots, D f_{\kappa}\left(x^{\prime}\right)$ are linearly independent. Then there exists a neighbourhood $U\left(x^{\prime}\right)$ such that $D f_{1}(x), \ldots, D f_{\kappa}(x), x \in U\left(x^{\prime}\right)$ are linearly independent. By Theorem 4.3, $f\left(U\left(x^{\prime}\right)\right)$ has a nonempty interior (see e.g. proof of (1) of Prop. 5.2) and $f\left(U\left(x^{\prime}\right)\right) \subset f\left(U\left(x_{0}\right)\right) \subset F^{-1}(0)$, hence $F^{-1}(0)$ would not be nowhere dense. 
The definition of functional dependence at $x_{0}$ given in Chapter 4 of [28] can be rewritten in Banach spaces as follows.

Definition 7.13. Let $E$ be a Banach space and let functions $f_{i}: U \rightarrow \mathbb{R}, U \subset E$ open, $i=1, \ldots, \kappa$ be of class $C^{1}$ in a neighbourhood of $x_{0} \in U$. Functions $f_{1} \ldots, f_{\kappa}$ are functionally dependent at $x_{0}$, if

$$
\operatorname{rank}\left\{D f_{i}\left(x_{0}\right), i=1, \ldots, \kappa\right\}<\kappa \text {. }
$$

Otherwise, we say that $f_{1} \ldots, f_{\kappa}$ are functionally independent at $x_{0}$.

Let $\Omega \subset U$ be an open set. We say that functions $f_{i}: U \rightarrow \mathbb{R}$, of class $C^{1}, i=1, \ldots, \kappa$, are functionally dependent on $\Omega$ if (7.1) holds for all $x_{0} \in \Omega$. Functions $f_{i}: U \rightarrow \mathbb{R}$, of class $C^{1}, i=1, \ldots, \kappa$, are functionally independent on $\Omega$ if

$$
\operatorname{rank}\left\{D f_{i}(x), i=1, \ldots, \kappa\right\}=\kappa \text { for all } x \in \Omega \text {. }
$$

In Theorem 4.1.3 of [28] it was shown that for $f_{i}: \mathbb{R}^{\kappa} \rightarrow \mathbb{R}, i=1, \ldots, \kappa$, Definition 7.5 and Definition 7.13 are equivalent.

Remark 7.14. Let $E$ be a Banach space. By Fact 7.3, if functions $f_{i}: U \rightarrow \mathbb{R}$, of class $C^{1}, i=1, \ldots, \kappa$, are functionally dependent at $x_{0} \in U, U \subset E$ open, in the sense of Definition 5.1, then there exists a neighbourhood $U\left(x_{0}\right)$ such that they are functionally dependent on $U\left(x_{0}\right)$ in the sense of Definition 7.13.

The following example illustrates the fact that the functional dependence in the sense of Definition 7.13 in a neighbourhood of $x_{0}$ does not imply the functional dependence at $x_{0}$ in the sense of Definition 5.1.

Example 7.15. Let us consider the geometric tornado function $f=\left[f_{1}, f_{2}, f_{3}\right], f_{1}, f_{2}, f_{3}: \mathbb{R} \rightarrow \mathbb{R}$ defined as follows (see Fig. 1)

$$
\begin{aligned}
& f_{1}(x)= \begin{cases}x^{3} \sin \left(\frac{1}{x}\right) & \text { if } x \neq 0, \\
0 & \text { otherwise, }\end{cases} \\
& f_{2}(x)= \begin{cases}x^{3} \cos \left(\frac{1}{x}\right) & \text { if } x \neq 0, \\
0 & \text { otherwise, }\end{cases} \\
& f_{3}(x)=x^{3} .
\end{aligned}
$$

We will show, that $f_{1}, f_{2}, f_{3}$ are functionally dependent in the sense of Definition 7.13 on any open set $\Omega$ which contains $x_{0}=0$ and are functionally independent at $x_{0}$ in the sense of Definition 5.1.

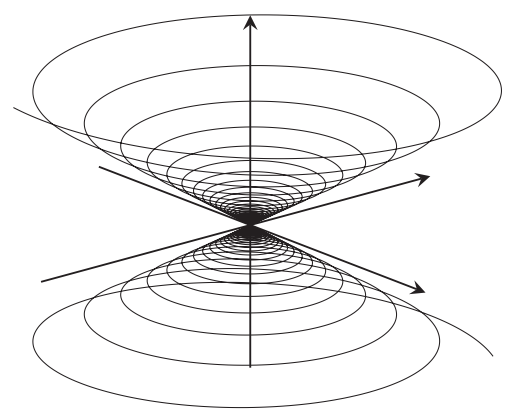

Figure 1 . The image of $\mathbb{R}$ under $f=\left[f_{1}, f_{2}, f_{3}\right]$. 
Derivatives of functions $f_{1}, f_{2}, f_{3}$ are as follows

$$
\begin{aligned}
& f_{1}^{\prime}(x)= \begin{cases}3 x^{2} \sin \left(\frac{1}{x}\right)-x \cos \left(\frac{1}{x}\right) & \text { if } x \neq 0, \\
0 & \text { otherwise }\end{cases} \\
& f_{2}^{\prime}(x)= \begin{cases}3 x^{2} \cos \left(\frac{1}{x}\right)+x \sin \left(\frac{1}{x}\right) & \text { if } x \neq 0, \\
0 & \text { otherwise }\end{cases} \\
& f_{3}^{\prime}(x)=3 x^{2} .
\end{aligned}
$$

For any open set $U_{0}$ containing $x_{0}=0$ we have $\operatorname{rank}\left\{f_{1}^{\prime}(x), f_{2}^{\prime}(x), f_{3}^{\prime}(x)\right\}<3, x \in U_{0}$. Hence, functions $f_{1}, f_{2}, f_{3}$ are functionally dependent on $U_{0}$ in the sense of Definition 7.13.

Let $U\left(x_{0}\right)$ be any neighbourhood of $x_{0}$ and let $F: \mathbb{R}^{3} \rightarrow \mathbb{R}$ be any $C^{1}$ function such that $F\left(f\left(U\left(x_{0}\right)\right)\right)=0$, where $f(x)=\left[f_{1}(x), f_{2}(x), f_{3}(x)\right]$. Let $y_{0}=f\left(x_{0}\right)=(0,0,0)$ and $t_{n}=\frac{1}{\frac{\pi}{2}+2 n \pi}$. Then

$$
F\left(t_{n}^{3}[1,0,1]\right)=F\left(t_{n}^{3}, 0, t_{n}^{3}\right)=F\left(f_{1}\left(t_{n}\right), f_{2}\left(t_{n}\right), f_{3}\left(t_{n}\right)\right)=0
$$

for sufficiently large $n$ and

$$
F^{\prime}\left(y_{0},[1,0,1]\right)=\lim _{t \rightarrow 0} \frac{F(t[1,0,1])}{t}=\lim _{n \rightarrow+\infty} \frac{F\left(t_{n}^{3}[1,0,1]\right)}{t_{n}^{3}}=\lim _{n \rightarrow+\infty} \frac{0}{t_{n}^{3}}=0 .
$$

Analogously one can show that

$$
F^{\prime}\left(y_{0},[0,1,1]\right)=0, \quad F^{\prime}\left(y_{0},[-1,0,1]\right)=0 .
$$

Hence $D F\left(y_{0}\right)=0$, i.e functions $f_{1}, f_{2}, f_{3}$ cannot be functionally dependent at $x_{0}$ in the sense of Definition 5.1.

Remark 7.16. Let $f=\left[f_{1}, \ldots, f_{\kappa}\right]: \mathbb{R}^{k} \rightarrow \mathbb{R}^{\kappa}$ be of class $C^{1}$ in a neighbourhood of $x_{0} \in \mathbb{R}^{k}$. If for any neighbourhood $U\left(x_{0}\right)$ there exist $v_{1}, \ldots, v_{\kappa} \in \mathbb{R}^{\kappa}$ linearly independent and a sequence $t_{m}>0, t_{m} \rightarrow 0$ such that $f\left(U\left(x_{0}\right)\right) \cap\left(f\left(x_{0}\right)+t_{m} v_{i}\right) \neq \emptyset$ for all $m \in \mathbb{N}$, then functions $f_{1}, \ldots, f_{\kappa}$ are functionally independent at $x_{0}$ in the sense of Definition 5.1.

It is clear that Proposition 7.4 implies Remark 7.16 and the converse does not hold (as in Exam. 7.15).

In conclusion, the established relationships between above concepts of functional dependence at $x_{0}$ are illustrated in Figure 2.

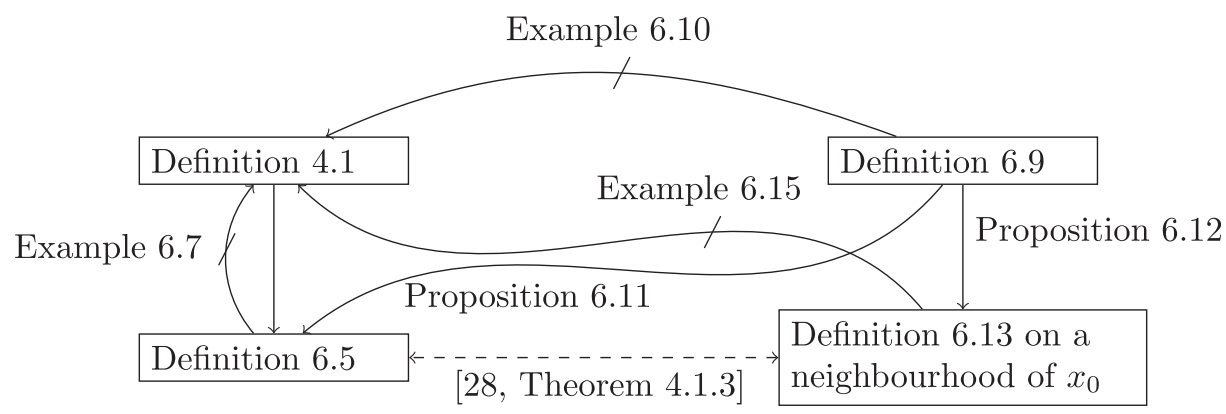

Figure 2. Relationships between functional dependence concepts. Theorem 4.1 .3 of [28] is given in the case $f_{1}, \ldots, f_{\kappa}: \mathbb{R}^{\kappa} \rightarrow \mathbb{R}$. 


\section{RELAXED CONSTANT RANK CONSTRAINT QUALIFICATION AND LAGRANGE MULTIPLIERS}

In this section we prove the non-emptiness of Lagrange multipliers set at a local minimum under RCRCQ and as an application we show that the linearized problem $(\mathrm{P})$ at a local minimum is solvable.

Let us consider the problem $(\mathrm{P})$,

$$
\begin{array}{ll}
\operatorname{minimize} & h_{0}(x) \\
\text { s.t. } & x \in \mathcal{F},
\end{array}
$$

where $\mathcal{F}$ is of the form (1.1), i.e.

$$
\mathcal{F}:=\left\{x \in E \mid h_{i}(x)=0, i \in I_{0}, h_{i}(x) \leq 0, i \in I\right\},
$$

where $E$ is a Banach space, $h_{0}, h_{i}: E \rightarrow \mathbb{R}, i \in I_{0} \cup I$ are of class $C^{1}$ in a neighbourhood of a given local minimum $x_{0} \in \mathcal{F}$ of $h_{0}$ and sets $I_{0}, I$ are finite $\left(I_{0} \cup I=\{1, \ldots, n\}\right)$.

Let $\Lambda(x)$ be the set of Lagrange multipliers at $x \in \mathcal{F}$, i.e.

$$
\Lambda(x):=\left\{\lambda \in \mathbb{R}^{n} \mid D_{x} L(\lambda, x)=0, \lambda_{i} \geq 0 \text { and } \lambda_{i} h_{i}(x)=0, i \in I\right\},
$$

where

$$
L(\lambda, x):=h_{0}(x)+\sum_{i=1}^{n} \lambda_{i} h_{i}(x), \quad \lambda=\left(\lambda_{1}, \ldots, \lambda_{n}\right)
$$

is the Lagrangean to problem (P). The following proposition relates RCRCQ to the existence of Lagrange multipliers. More on this topic see [8].

Proposition 8.1. Let $x_{0} \in \mathcal{F}$ be a local minimum of problem $(\mathrm{P})$ and let $R C R C Q$ hold for $\mathcal{F}$ at $x_{0}$. Then $\Lambda\left(x_{0}\right) \neq \emptyset$.

Proof. By Theorem 6.5, for any $d \in \Gamma_{\mathcal{F}}\left(x_{0}\right)$ there exists a vector function $o(t)$ such that $\lim _{t \rightarrow 0}\|o(t)\| t^{-1}=0$ and $x_{0}+t d+o(t) \in \mathcal{F}$. Since $x_{0}$ is a local minimum of $h_{0}$ on $\mathcal{F}$ we have $h_{0}\left(x_{0}+t d+o(t)\right)-h_{0}\left(x_{0}\right) \geq 0$ for all $t$ sufficiently small. By Taylor expansion, we have

$$
\begin{aligned}
0 & \leq h_{0}\left(x_{0}+t d+o(t)\right)-h_{0}\left(x_{0}\right) \\
& =h_{0}\left(x_{0}\right)+\left\langle D h_{0}\left(x_{0}+\theta(t d+o(t))\right), t d+o(t)\right\rangle-h_{0}\left(x_{0}\right) \\
& =t\left\langle D h_{0}\left(x_{0}+\theta(t d+o(t))\right), d\right\rangle+\left\langle D h_{0}\left(x_{0}+\theta(t d+o(t))\right), o(t)\right\rangle,
\end{aligned}
$$

where $\theta \in[0,1]$ and $\theta$ depends on $t, d$. Hence,

$$
\left\langle D h_{0}\left(x_{0}+\theta(t d+o(t))\right), d\right\rangle \geq-\left\langle D h_{0}\left(x_{0}+\theta(t d+o(t))\right), o(t) t^{-1}\right\rangle .
$$

By passing to the limit with $t \rightarrow 0$ in (8.2) we obtain $\left\langle D h_{0}\left(x_{0}\right), d\right\rangle \geq 0$. Hence

$$
-D h_{0}\left(x_{0}\right) \in\left(\Gamma_{\mathcal{F}}\left(x_{0}\right)\right)^{\circ},
$$

where $\left(\Gamma_{\mathcal{F}}\left(x_{0}\right)\right)^{\circ}$ is dual cone defined as

$$
\left(\Gamma_{\mathcal{F}}\left(x_{0}\right)\right)^{\circ}:=\left\{d^{*} \in E^{*} \mid\left\langle d^{*}, d\right\rangle \leq 0, \forall d \in \Gamma_{\mathcal{F}}\left(x_{0}\right)\right\} .
$$


Since

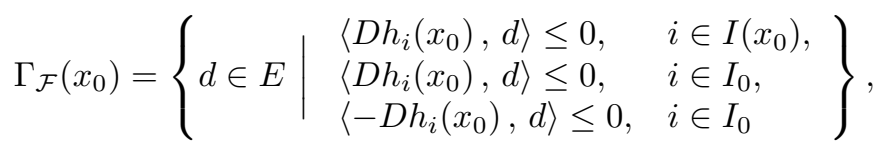

by Theorem 6.40 of [13], the dual cone to $\Gamma_{\mathcal{F}}\left(x_{0}\right)$ is given as follows

$$
\begin{aligned}
\left(\Gamma_{\mathcal{F}}\left(x_{0}\right)\right)^{\circ}=\left\{d^{*} \in E^{*} \mid d^{*}=\right. & \sum_{i \in I_{0} \cup I\left(x_{0}\right)} \lambda_{i} D h_{i}\left(x_{0}\right), \\
& \left.\lambda_{i} \geq 0, i \in I\left(x_{0}\right), \quad \lambda_{i} \in \mathbb{R}, \quad i \in I_{0}\right\} .
\end{aligned}
$$

By (8.3) and (8.4), there exist $\lambda_{i} \geq 0, i \in I\left(x_{0}\right), \lambda_{i} \in \mathbb{R}, i \in I_{0}$ such that

$$
-D h_{0}\left(x_{0}\right)=\sum_{i \in I_{0} \cup I\left(x_{0}\right)} \lambda_{i} D h_{i}\left(x_{0}\right) .
$$

By putting $\lambda_{i}=0$ for $i \in\{1,2, \ldots, n\} \backslash\left(I_{0} \cup I\left(x_{0}\right)\right)$, we have $\Lambda\left(x_{0}\right) \neq \emptyset$.

As an application of Proposition 8.1 we show that the linearized problem, at a local minimum to (P), $x_{0} \in \mathcal{F}$,

$$
\begin{array}{ll}
\operatorname{minimize} & \left\langle D h_{0}\left(x_{0}\right), d\right\rangle \\
\text { s.t. } & \left\langle D h_{i}\left(x_{0}\right), d\right\rangle \leq 0, \quad i \in I\left(x_{0}\right), \\
& \left\langle D h_{i}\left(x_{0}\right), d\right\rangle=0, \quad i \in I_{0}
\end{array}
$$

is solvable with a solution $d=0$. Problem (8.5) can be equivalently rewritten as

$$
\begin{array}{ll}
\operatorname{minimize} & \left\langle D h_{0}\left(x_{0}\right), d\right\rangle \\
\text { s.t. } & \left\langle D h\left(x_{0}\right), d\right\rangle \in K,
\end{array}
$$

where $h=\left[h_{i}\right]_{i \in I\left(x_{0}\right) \cup I_{0}}, K=\left\{k=\left(k_{i}\right) \in \mathbb{R}^{\left|I_{0} \cup I\left(x_{0}\right)\right|} \mid k_{i} \leq 0, i \in I\left(x_{0}\right), k_{i}=0, i \in I_{0}\right\}$. Lagrangian to (8.6) is defined as follows

$$
L(d, \lambda)=\left\langle D h_{0}\left(x_{0}\right), d\right\rangle+\left\langle\lambda, D h\left(x_{0}\right) d\right\rangle
$$

where $\lambda \in \mathbb{R}^{\left|I_{0} \cup I\left(x_{0}\right)\right|}$. Let $K^{*}:=\left\{k^{*}=\left(k_{i}^{*}\right) \in \mathbb{R}^{\left|I_{0} \cup I\left(x_{0}\right)\right|} \mid\left\langle k, k^{*}\right\rangle \leq 0\right.$ for all $\left.k \in K\right\}=\left\{k^{*} \in \mathbb{R}^{\left|I_{0} \cup I\left(x_{0}\right)\right|} \mid k_{i}^{*} \geq\right.$ $\left.0, i \in I\left(x_{0}\right), k_{i}^{*} \in \mathbb{R}, i \in I_{0}\right\}$. The dual to (8.6) takes the form

$$
\begin{array}{ll}
\operatorname{maximize} & \inf _{d \in E} L(d, \lambda) \\
\text { s.t } & \lambda \in K^{*} .
\end{array}
$$

Let us consider the objective of the dual. We have

$$
\begin{aligned}
\inf _{d \in E} L(d, \lambda) & =\inf _{d \in E}\left\{\left\langle D h_{0}\left(x_{0}\right), d\right\rangle+\left\langle\lambda, D h\left(x_{0}\right) d\right\rangle\right\} \\
& =\inf _{d \in E}\left\{\left\langle D h_{0}\left(x_{0}\right), d\right\rangle+\sum_{i \in I_{0} \cup I\left(x_{0}\right)} \lambda_{i}\left\langle D h_{i}\left(x_{0}\right), d\right\rangle\right\}
\end{aligned}
$$




$$
=\inf _{d \in E}\left\{\left\langle D h_{0}\left(x_{0}\right)+\sum_{i \in I_{0} \cup I\left(x_{0}\right)} \lambda_{i} D h_{i}\left(x_{0}\right), d\right\rangle\right\} .
$$

Hence,

$$
\begin{aligned}
\inf _{d \in E}\left\langle D h_{0}\left(x_{0}\right)\right. & \left.+\sum_{i \in I_{0} \cup I\left(x_{0}\right)} \lambda_{i} D h_{i}\left(x_{0}\right), d\right\rangle \\
& = \begin{cases}-\infty & \text { if } D h_{0}\left(x_{0}\right)+\sum_{i \in I_{0} \cup I\left(x_{0}\right)} \lambda_{i} D h_{i}\left(x_{0}\right) \neq 0, \\
0 & \text { if } D h_{0}\left(x_{0}\right)+\sum_{i \in I_{0} \cup I\left(x_{0}\right)} \lambda_{i} D h_{i}\left(x_{0}\right)=0 .\end{cases}
\end{aligned}
$$

Thus (8.8) is equivalent to the following

$$
\begin{array}{ll}
\operatorname{maximize} & 0 \\
\text { s.t } & D h_{0}\left(x_{0}\right)+\sum_{i \in I_{0} \cup I\left(x_{0}\right)} \lambda_{i} D h_{i}\left(x_{0}\right)=0, \\
& \lambda \in K^{*} .
\end{array}
$$

In conclusion, we obtain the following corollary.

Corollary 8.2. Under assumption of $R C R C Q$ at $x_{0} \in \mathcal{F}$, where $x_{0}$ is a local minimum of $(\mathrm{P})$, the element $d=0$ is a solution of (8.5), since, by Proposition 8.1, feasible set of (8.9) is nonempty.

Acknowledgements. We would like to thank Professor Krzysztof Kurdyka and anonymous referees for their remarks and criticism which considerably improved the presented results.

\section{REFERENCES}

[1] J. Abadie, On the Kuhn-Tucker theorem, in Nonlinear Programming (NATO Summer School, Menton, 1964). North-Holland, Amsterdam (1967) 19-36.

[2] R. Abraham, J.E. Marsden and T. Ratiu, Manifolds, tensor analysis, and applications. Vol. 75 of Applied Mathematical Sciences, second ed. Springer-Verlag, New York (1988).

[3] R. Andreani, R. Behling, G. Haeser and P.J.S. Silva, On second-order optimality conditions in nonlinear optimization. Optim. Methods Softw. 32 (2017) 22-38.

[4] R. Andreani, C.E. Echagüe and M.L. Schuverdt, Constant-rank condition and second-order constraint qualification. J. Optim. Theory Appl. 146 (2010) 255-266.

[5] R. Andreani, N.S. Fazzio, M.L. Schuverdt and L.D. Secchin, A sequential optimality condition related to the quasi-normality constraint qualification and its algorithmic consequences. SIAM J. Optim. 29 (2019) 743-766.

[6] R. Andreani, G. Haeser, M.L. Schuverdt and P.J.S. Silva, A relaxed constant positive linear dependence constraint qualification and applications. Math. Program. 135 (2012) 255-273.

[7] R. Andreani and P. Silva, Constant rank constraint qualifications: a geometric introduction. Pesquisa Operacional 34 (2014) 481-494.

[8] E.M. Bednarczuk, L.I. Minchenko and K.E. Rutkowski, On lipschitz-like continuity of a class of set-valued mappings. Optimization 62 (2019) 2535-2549.

[9] R. Bergmann and R. Herzog, Intrinsic formulation of KKT conditions and constraint qualifications on smooth manifolds. Preprint arXiv:1804.06214 (2018).

[10] J.F. Bonnans and A. Shapiro, Perturbation analysis of optimization problems. Springer Series in Operations Research. Springer-Verlag, New York (2000).

[11] W.M. Boothby, An introduction to differentiable manifolds and Riemannian geometry. Vol. 120 of Pure and Applied Mathematics, second ed. Academic Press, Inc., Orlando, FL (1986).

[12] E. Börgens, C. Kanzow, P. Mehlitz and G. Wachsmuth, New Constraint Qualifications for Optimization Problems in Banach Spaces based on Cone Continuity Properties. Preprint arXiv:1912.06531 (2019).

[13] F. Deutsch, Best approximation in inner product spaces. Vol. 7 of CMS Books in Mathematics/Ouvrages de Mathématiques de la SMC. Springer-Verlag, New York (2001).

[14] J. Gallier and J. Quaintance, Linear Algebra and Optimization with Applications to Machine Learning: Volume I: Linear Algebra for Computer Vision, Robotics, and Machine Learning. World Scientific Publishing Co Pte Ltd (2020).

[15] R. Henrion and J.V. Outrata, Calmness of constraint systems with applications. Math. Program. 104 (2005) $437-464$. 
[16] A.D. Ioffe and V.M. Tihomirov, Theory of extremal problems. Vol. 6 of Studies in Mathematics and its Applications. Translated from the Russian by Karol Makowski. North-Holland Publishing Co., Amsterdam-New York (1979).

[17] R. Janin, Directional derivative of the marginal function in nonlinear programming. Springer Berlin Heidelberg, Berlin, Heidelberg (1984) 110-126.

[18] A.Y. Kruger, L. Minchenko and J.V. Outrata, On relaxing the mangasarian-fromovitz constraint qualification. Positivity 18 (2014) 171-189.

[19] S. Kurcyusz, On the existence and nonexistence of lagrange multipliers in Banach spaces. J. Optim. Theory Appl. 20 (1976) 81-110.

[20] W. Li, Abadie's constraint qualification, metric regularity, and error bounds for differentiable convex inequalities. SIAM J. Optim. 7 (1997) 966-978.

[21] K. Maurin, Analysis. Part I. Elements, Translated from the Polish by Eugene Lepa. D. Reidel Publishing Co., DordrechtBoston, Mass.; PWN-Polish Scientific Publishers; Warsaw (1976).

[22] L. Minchenko, Note on Mangasarian-Fromovitz-like constraint qualifications. J. Optim. Theory Appl. 182 (2019) 1199-1204.

[23] L. Minchenko and S. Stakhovski, On relaxed constant rank regularity condition in mathematical programming. Optimization 60 (2011) 429-440.

[24] R. Narasimhan, Lectures on topics in analysis, Notes by M. S. Rajwade. Tata Institute of Fundamental Research Lectures on Mathematics, No. 34, Tata Institute of Fundamental Research, Bombay (1965).

[25] J.-P. Penot, On the existence of lagrange multipliers in nonlinear programming in banach spaces, in Optimization and Optimal Control, edited by A. Auslender, W. Oettli, and J. Stoer. Springer Berlin Heidelberg (1981) 89-104.

[26] S.M. Robinson, Stability theory for systems of inequalities. II. Differentiable nonlinear systems. SIAM J. Numer. Anal. 13 (1976) 497-513.

[27] M. Solodov, Constraint Qualifications. Wiley Encyclopedia of Operations Research and Management Science (2011).

[28] L. Székelyhidi, Ordinary and partial differential equations for the beginner. World Scientific Publishing Co. Pte. Ltd., Hackensack, NJ (2016).

[29] V.A. Zorich, Mathematical analysis. I, Universitext, second ed. Translated from the 6th corrected Russian edition, Part I, 2012 by Roger Cooke, With Appendices A-F and new problems translated by Octavio Paniagua T. Springer-Verlag, Berlin (2015).

[30] J. Zowe and S. Kurcyusz, Regularity and stability for the mathematical programming problem in Banach spaces. Appl. Math. Optim. 5 (1979) 49-62. 